\title{
Flow Separation Dynamics in Three-Dimensional Asymmetric Diffusers
}

\author{
Arthur Hajaali $^{1} \cdot$ Thorsten Stoesser $^{1}$ (D)
}

Received: 13 January 2021 / Accepted: 9 November 2021 / Published online: 19 November 2021

(C) The Author(s) 2021

\begin{abstract}
The mean and instantaneous flow separation of two different three-dimensional asymmetric diffusers is analysed using the data of large-eddy simulations. The geometry of both diffusers under investigation is based on the experimental configuration of Cherry et al. (Int J Heat Fluid Flow 29(3):803-811, 2008). The two diffusers feature similar area ratios of $\mathrm{AR}=4.8$ and $\mathrm{AR}=4.5$ while exhibiting differing asymmetric expansion ratios of $\mathrm{AER}=4.5$ or AER $=2.0$, respectively. The Reynolds number based on the averaged inlet velocity and height of the inlet duct is approximately $R e=10,000$. The time-averaged flow in both diffusers in terms of streamwise velocity profiles or the size and location of the mean backflow region are validated using experimental data. In general good agreement of simulated results with the experimental data is found. Further quantification of the flow separation behaviour and unsteadiness using the backflow coefficient reveals the volume portion in which the instantaneous reversal flow evolves. This new approach investigates the cumulative fractional volume occupied by the instantaneous backflow throughout the simulation, a power density spectra analysis of their time series reveals the periodicity of the growth and reduction phases of the flow separation within the diffusers. The dominating turbulent events responsible for the formation of the energy-containing motions including ejection and sweep are examined using the quadrant analysis at various locations. Finally, isourfaces of the Q-criterion visualise the instantaneous flow and the origin and fate of coherent structures in both diffusers.
\end{abstract}

Keywords Large eddy simulation · Flow separation unsteadiness · Three-dimensional asymmetric diffuser $\cdot$ Coherent-structures

\section{Introduction}

A diffuser is a gradual or abrupt expansion or enlargement of the cross-sectional area of a duct, pipe or canal whose main purpose is to reduce a fluid flow's kinetic energy. Diffusers are ubiquitous in engineering applications for instance in pipe networks of the water and the ventilation industry (Laliberte et al. 1983). They are also often succeeding

Thorsten Stoesser

t.stoesser@ucl.ac.uk

1 University College London, London, UK 
combustion chambers (Fishenden and Stevens 1977) in jet engines in the aeronautics and automotive industry. Diffusers are used in the form of draft tubes of hydro turbines such as bulb (Duquesne et al. 2016), Kaplan (Daniels et al. 2020) or Francis turbines (Zhang et al. 2009). The industry's infatuation with optimising diffuser designs with the goal to minimise energy losses has led to a sea of experimental research in recent decades with the goal to further understand the key parameters affecting the diffuser efficiency. Multiple geometrical parameters such as cross-section geometry (Gibson 1910), aspect ratio, area ratio (Welsh 1976), length of the tail section (Kline 1959) or curvature of the diffuser wall (Patterson 1938) have been examined. A diffuser's main purpose is to reduce the incoming kinetic energy (velocity) converting the flow's dynamic pressure into static pressure. A too abrupt conversion of these pressures leads to flow separation and to energy losses in the system which is to be avoided in most applications.

Flow separation occurs under various flow conditions and environments, however mainly when the flow slows down (e.g. due to a sudden expansion of the geometry) and hence pressure increases (following Bernoulli's principle) in the streamwise direction, i.e. a so-called adverse pressure gradient. In diffusers, sharp corners can trigger flow separation when viscous forces within the boundary layer near the diffuser wall are overcome by the fluid's momentum forces leading to local detachment of the fluid from the boundary. In turbulent flows, separation leads to the development of coherent turbulent structures and increased dynamic pressure disturbing the reduction in static pressure. The diffuser's expansion generates strong adverse pressure gradients acting against the streamwise velocity of the turbulent boundary layer, and once the velocity reduces to zero, flow detachment takes place. The backflow within the flow separation region acts as an obstructing volume inside the diffuser leading to local acceleration of the flow which affects negatively the diffuser's performance. The separation of the turbulent boundary layer is highly unsteady and three-dimensional in nature challenging both design engineers and the research community in understanding and classifying fluid flows in diffusers.

The lack of sophisticated and non-invasive experimental instrumentation and limited computational resources initially asked researchers to simplify the complexity of the flow separation phenomenon by investigating quasi two-dimensional diffusers. Obi et al. (1993) first experimentally (LDV) and then numerically (RANS) investigated the mean flow field of a $10^{\circ}$ planar diffuser the flow of which is governed by a distinctive flow separation bubble. Subsequently, Obi et al. (1999) provoked an artificial perturbation at the beginning of the diffuser and demonstrated its impact on the detachment and reattachment location of the mean flow separation. Wu et al.'s numerical study (2006) provides the structural features of the internal layer located at the flat wall of the diffuser, it is generated by the low-frequency of the turbulent fluctuation. Another planar diffuser with $8^{\circ}$ diverging angle was researched by Törnblom et al. (2009) in which the time-averaged flow field was monitored and the energy spectra, instantaneous flow and auto-correlations were analysed to reveal the existence of large-scale hairpin vortices. This experimental study subsequently led Herbst et al. (2007) to studying the influence of the Reynolds number on the mean-flow separation size and location. The results suggest that the boundary layer has less tendency to separate at the diffuser throat when the Reynolds number is high.

Cherry et al. (2008) was the first to develop an experimental methodology to measure accurately the hydrodynamics within two different three-dimensional asymmetric diffusers. This experiment successfully monitored the development and behaviour of a three-dimensional flow separation induced by a strong adverse pressure gradient. The expansion part of their first diffuser (D1) and their second diffuser (D2) had the same duct inlet geometry while featuring a different outlet geometry (D1 and D2 are depicted in Fig. 1). A medical 


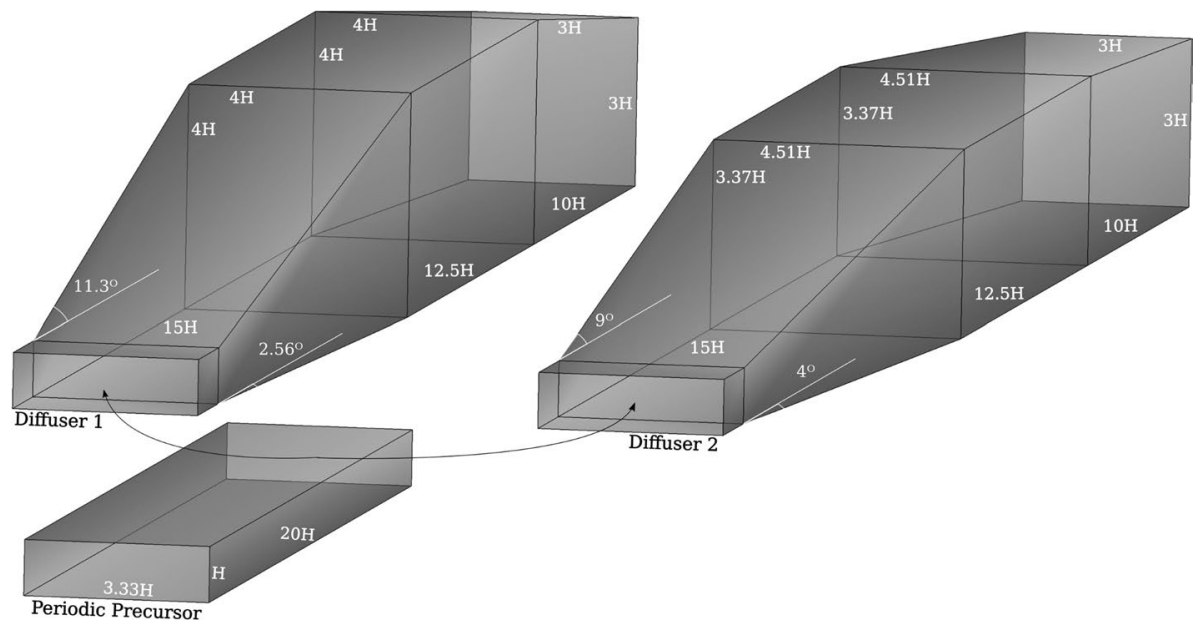

Fig. 1 Geometry and dimensions of the precursor channel and the two asymmetric rectangular diffusers D1 and D2

magnetic resonance velocimetry (MRV) method monitored and collected magnetic resonance signals which were then post-processed and transformed into a velocity field. This non-intrusive method provided accurate results of the mean velocity field $( \pm 5 \%)$ and its mean fluctuation $( \pm 10 \%)$. The data set has been instrumental towards the calibration and validation of numerous computational methods and models. Cherry et al. (2010) later investigated the flow field and performance of an annular diffuser consisting of two different inlets, one had a fully developed flow while the other inlet was a wake induced by a row of struts. Cherry et al.'s experiment (2008) laid the groundwork for Grundmann et al.'s investigation which induced a different inlet secondary current using a plasma actuator and observed a significant difference in the mean flow-separation size and location which led to an improvement of the pressure recovery of 13-17\% (Grundmann et al. 2012).

The first CFD study aiming to reproduce the hydrodynamics in the two diffusers of Cherry et al. (2008) was carried out by Schneider et al. (2010), who performed a grid sensitivity analysis from multiple RANS and LES simulations investigating the time-averaged flow field of both D1 and D2. The RANS simulations failed in predicting accurately the location and size of the reverse flow region while the LES results on a fine grid were in good agreement with experimental data. Other studies including Abe and Ohtsuka (2010) and Jakirlić et al. (2010) compared the accuracy of hybrid LES/RANS (HLR) models with pure LES in terms of the mean flow separation within the first diffuser D1. These papers revealed that HLR is able to deliver similar results to LES at reduced computational cost as HLR simulations were run on coarser meshes. Ohlsson et al. (2010) validated their DNS of one of the diffuser flows of Cherry et al. (2008) in terms of turbulence statistics. In a second paper Malm et al. (2012) the authors furthered the understanding of the flow separation unsteadiness and the quasi-periodic meandering of the core flow using the backflow coefficient and a power-spectral analysis while revealing coherent structures inherent to flow separation through a POD investigation.

The present paper investigates the influence of the aspect ratio on the development and the hydrodynamic behaviour of the flow separation within two geometrically similar asymmetric rectangular diffusers. The time-averaged flow is cross-validated with experimental 
and DNS data, subsequently, the difference of the flow separation and reverse flow in the two diffusers is assessed. A methodology evaluating the Power Spectral Density of the cumulative flow reversal volume within the diffuser is introduced. This analysis further characterises the flow separation intermittency by capturing its growth and reduction frequencies. The typology and location of coherent structure motions responsible for the detachment and separation of the flow is identified using the $\mathrm{Q}$ criterion and quadrant analysis.

\section{Numerical Framework}

\subsection{Large-eddy Simulation Code}

The simulations are performed with Hydro3D, an open-source LES code (https://github. com/OuroPablo/Hydro3D) that discretises the governing filtered Navier-Stokes equations (Eq. 1) using a finite-difference method on a Cartesian staggered grid. The filtering is solely based on the volume of the fluid cell $\Delta=(\Delta x \Delta y \Delta z)^{1 / 3}$. The scales of motion larger than the filter cutoff are resolved through the descritisation of the partial differential equations while the smaller scales are accounted for by a sub-grid scale (SGS) model. Hydro3D has undergone thorough validation for several industrial flow conditions including openchannel flows (Bomminayuni and Stoesser 2011; Stoesser et al. 2015), free-surface flows (McSherry et al. 2017, 2018), or hydrodynamics of hydrokinetic turbines (Ouro and Stoesser 2017, 2019). However, here validation for internal separated flows in asymmetric diffusers is preceeding further data analysis. The governing equations are written as:

$$
\begin{aligned}
& \frac{\partial\left(u_{i}\right)}{\partial x_{i}}=0 \\
& \frac{\partial\left(u_{i}\right)}{\partial t}+\frac{\partial\left(u_{i} u_{j}\right)}{\partial x_{j}}=-\frac{\partial p}{\partial x_{i}}+v \frac{\partial^{2} u_{i}}{\partial x_{i} x_{j}}-\frac{\partial \tau_{i j}}{\partial x_{j}}+f_{i}
\end{aligned}
$$

where $u_{i}, u_{j}(\mathrm{i}, \mathrm{j}, \mathrm{k}=1,2,3)$ is the filtered velocity vector in the three spatial directions $(\mathrm{x}, \mathrm{y}$, $\mathrm{z}$ ) stored at their respective cell faces while $p$ is the filtered pressure which is stored in the centre of the fluid cell. The geometry of the rectangular diffuser is represented by organised scatter of Immersed Boundary Points (IBP), the term $f_{i}$ corresponds to the external forces applied by the direct forcing Immersed Boundary Method to ensure no-slip condition at every IBP (Uhlmann 2005). $\tau_{i j}$ is the stress tensor resulting from filtering and represents the unresolved small-scale, or subgrid-scale (SGS) motion. The SGS stresses are approximated using the standard wall-adapting local eddy (WALE) viscosity model as originally proposed by Nicoud and Ducros (1999). This model implicitly calculates the SGS viscosity to provide an accurate dissipation. The WALE model improves its tensor framework in combining both the resolved strain rate and the resolved rotational rate, accounting for the possibility of $\nabla \mathbf{U} \neq 0$. In contrast to the Smagorinsky model, the WALE method does not require any damping near solid walls. This enables accurate predictions of the subgrid scale viscosity near solid surfaces, providing a clear advantage when using it in conjunction with the Immersed Boundary Method Kara et al. (2015), in which the grid does not follow solid surfaces. The fourth order central differencing schemes discretise the spatial first and second order derivatives (convection and diffusion terms) of the governing equation (Eq. 1) as described in Cevheri et al. (2016). Advancement in time is achieved through 
the fractional-step method (Chorin 1968). It is a predictor-corrector method that predicts the intermediate non-divergence free velocity field using a two step Runge-Kutta scheme before projecting these velocities onto a divergence-free vector field using the Poisson equation. Subsequently, the Poisson equation is solved using the Strongly Implicit Procedure (SIP) fully detailed in Azevedo et al. (1988).

\subsection{Simulation Set-Up}

The geometrical set-up of the two diffusers under consideration, namely D1 and D2, is nearly identical to the geometry used in the laboratory experiment carried out by Cherry et al. (2008). The experimental geometry contains rounded corners while the numerical possess sharp corners for simplicity similar to Schneider et al. (2010), however due to the use of immersed boundaries to represent the geometry the corners are not as sharp as for a body-fitted grid. The two diffusers are composed of four distinctive parts which are illustrated in Fig. 1: (a) first an inlet duct $(x / H \in[-2,0])$, second the expansion part $(x / H \in[0,15])$, the third part is the extension or tail section $(x / H \in[15,27.5])$ and finally the outlet section $(x / H \in[27.5,37.5])$. Figure 1 provides the detailed geometry of both diffusers, the only difference between diffusers D1 and D2 is the aspect ratio of the outlet cross-section of the expansion part of the diffuser, respectively featuring aspect ratios ( $A S=h / b$, i.e. the height-to-width-ratio) of 1:1 (D1) or 1:1.34 (D2), respectively. This can be translated into a more comprehensive ratio, the asymmetric expansion ratio $(A E R)$ which describes the ratio of vertical expansion rate to the spanwise expansion rate. The diffusers D1 and D2 possess an asymmetric expansion ratio of $A E R_{D 1}=4.46$ or $A E R_{D 2}=2$, respectively for which significant differences in the development and behaviour of the flow separation is expected.

The Reynolds number of the inlet flows is $R e=10,000$, based on the average bulk velocity $U_{b}=1.0 \mathrm{~m} / \mathrm{s}$ in the inflow duct and its height $h=0.01 \mathrm{~m}$.

For the purpose of a fully developed inflow at low computational cost a precursor periodic simulation of the inlet duct is performed separately. A periodic boundary condition in the streamwise direction is used which provides a fully-developed flow field to be provided as inflow conditions into the diffuser domains. As Fig. 2 suggests first and second order
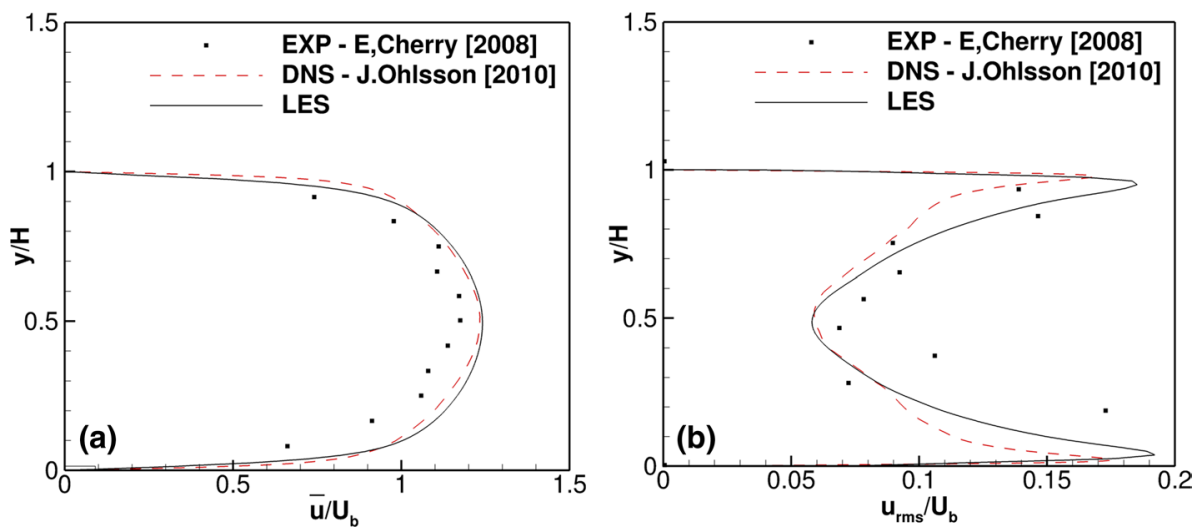

Fig. 2 Profiles of $\mathbf{a}$ Mean streamwise velocity and $\mathbf{b}$ Root mean square of streamwise fluctuation in the centre line of the inlet plane 
statistic in the converged precursor simulation are in convincing agreement with the experimental data of Cherry et al. (2008) and the DNS of Ohlsson et al. (2010). Both LES and DNS overestimate slightly the velocity in the centre of the duct, probably because the computational inlet ducts are infinitely long while the experimental inlet duct is rather short. The distribution of streamwise turbulent fluctuations is similar for LES and DNS whereas the experimental data is a bit more scattered, however agreement with the experiment is generally quite good. In order to provide a fully developed inflow inflow into the main diffuser domain planes of the instantaneous flow are continuously saved at every time step from the precursor simulation and provide instantaneous velocities $(\mathrm{u}, \mathrm{v}, \mathrm{w})$ at the inlet cross-section of the diffuser. A total of 20,000 time steps are saved corresponding to three flow through passages (FTP) of the entire length of the inlet duct.

The two simulations are performed with the same mesh resolution detailed in Table 1 providing the grid spacing $\left(\Delta_{x i}\right)$ and the number of grid points $\left(n_{x i}\right)$ in the three spatial directions, the number of elements of the computational domain $\left(N_{E}\right)$ and the number of elements located within the diffuser flow field $\left(N_{D E}\right)$. A grid sensitivity is performed during the calibration of the D1 but is not shown for brevity. Coarser simulations are ran to test different SGS models such as Smagorinsky or the turbulent subgrid scale energy one-equation model including $(\Delta x=0.015, \Delta y=0.01, \Delta z=0.01)$ and $(\Delta x=0.01, \Delta y=0.0005, \Delta z=0.0005)$ meshes but on coarser grids the detachment of the flow separation occurs too early near the throat of the diffuser leading to large over-prediction of the mean flow separation bubble. The corresponding wall unit for the refined mesh (Table 1) used for this study are $\Delta x^{+}=3.2, \Delta y^{+}=1.5, \Delta z^{+}=1.5$ which is very similar to the LES mesh of Jakirlić et al. (2010). In Fig. 3 the flow's power spectral density (PSD) is plotted for two distinct locations inside D1 (locations P4 and P8 as indicated in Fig. 19). P4 is inside the boundary near the straight wall and P8 is in the middle of the outlet channel. The thin black line represents the $-5 / 3$ Kolmogorow Law along which homogeneous turbulence decays. Whereas the velocity spectrum at P8 follows the $-5 / 3$ law quite well, the energy transfer in the boundary layer is not as steep, which might be expected given the anisotropy of turbulence near solid walls. Nevertheless, the LES is able to reproduce the transfer of energy from large scales to smaller scales over at least two decades in erms of the PSD and over at least one decade in terms of frequencies. At frequencies beyond $300 \mathrm{~Hz}$ the sgs-model kicks in and drains rather rapidly the energy from the flow. The

Fig. 3 Power spectral density (PSD) at two selected locations (Fig. 19) within the diffuser D1

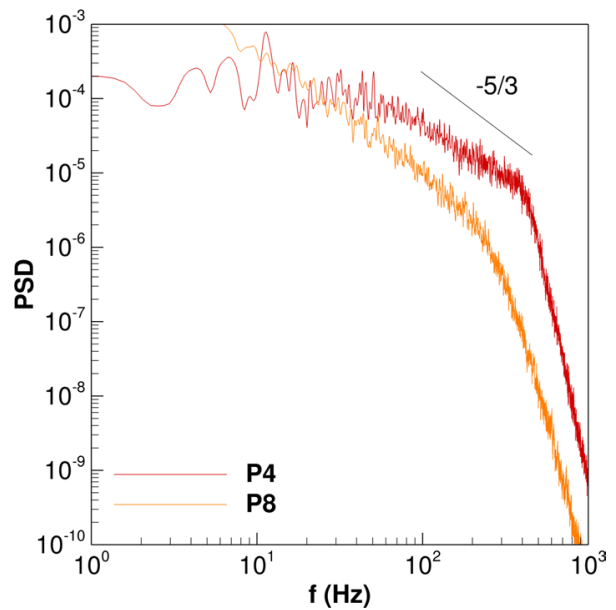




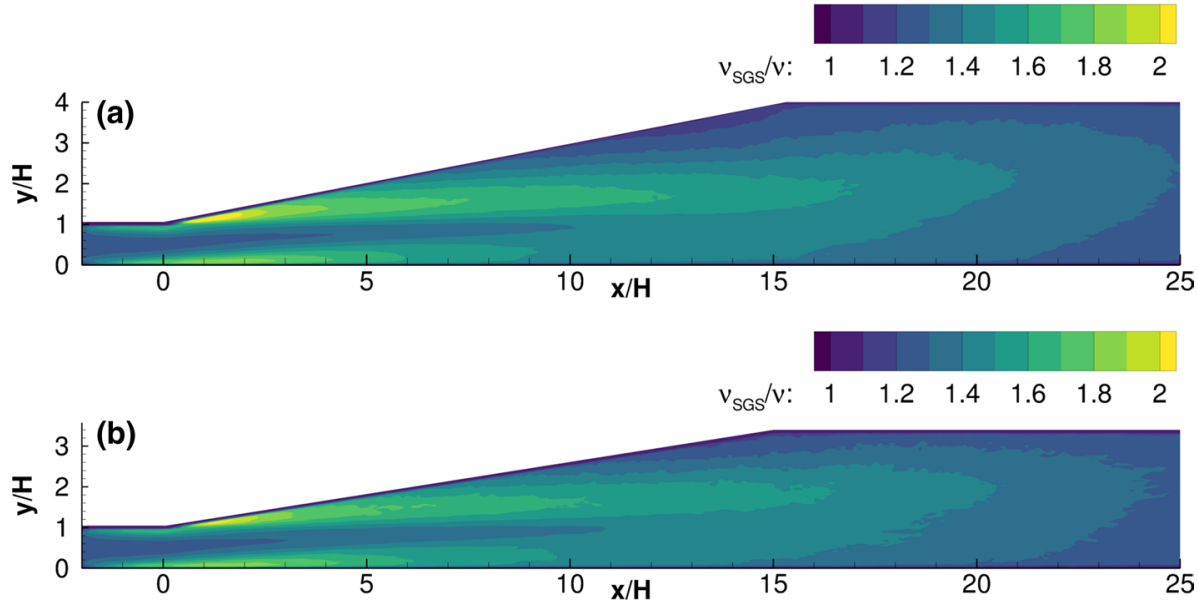

Fig. 4 Contours of the ratio of sub-grid scale viscosity to fluid kinematic viscosity for a D1, and b D2

Table 1 Mesh resolution of the different computational domains

\begin{tabular}{lllllllll}
\hline Sim & $\Delta x$ & $\Delta y$ & $\Delta z$ & $n_{x}$ & $n_{y}$ & $n_{z}$ & $N_{e}\left(10^{6}\right)$ & $N_{D e}\left(10^{6}\right)$ \\
\hline Precursor & 0.0005 & 0.000125 & 0.00025 & 200 & 320 & 272 & 10.24 & 3.46 \\
Diffuser 1 & 0.0005 & 0.000125 & 0.00025 & 800 & 320 & 160 & 40.96 & 30.18 \\
Diffuser 2 & 0.0005 & 0.000125 & 0.00025 & 800 & 272 & 182 & 39.60 & 28.93 \\
\hline
\end{tabular}

velocity spectra further the confidence in the grid and SGS model selection demonstrating realistic decay of turbulence and energy dissipation. Figure 4 presents contours of the ratio of subgrid-scale-viscosity-to-molecular viscosity $\left(v_{\text {sgs }} / v\right)$ demonstrating the small influence of subgrid-scale stresses on the flow. Only near the flow separation and in the shear layer are elevated levels of $v_{s g s} / v$ observed, and in general sgs-viscosity is less than twice the molecular viscosity, suggesting that large-scale turbulence is fully resolved by the simulation. The WALE model tends to return lower sgs-viscosities than the Smagorinsky model however and $v_{s g s}$ approaches zero near the immersed boundaries (in the expansion), as desired.

The computational domain is divided into 400 sub-domains for the D1 simulation and 384 sub-domains for the D2 simulation. Each sub-domain is surrounded by two layers of ghost-cells containing the flow information of the neighbouring sub-domain herein enabling the numerical approximation of the derivatives at its boundaries. The ghost-cell approach in conjunction with the Message Passing Interface (MPI) provides an effective communication between the different sub-domains of the entire computational domain (Ouro et al. 2019). The simulations are carried out on the Supercomputing Wales cluster using 200 Intel Xeon Gold 6148 cores for 188 hours, which corresponds to 37,600 CPU hours. The numerical stability is ensured with a fixed CFL $=0.5$ and a variable time step which averages to $d t_{a v}=6.7 \times 10^{-5}$ for D1 and $d t_{a v}=7.1 \times 10^{-5}$ for D2. The flow through passage (FTP) is calculated using of the mean cross-section area and mean velocity of each section of the diffusers, $F T P_{D 1}=1.44 s$ and $F T P_{D 2}=1.38 s$. The averaged flow field 
is collected for 25 FTPs while the time-averaged fluctuations are collected for 35 FTPs to ensure the convergence of the flow field within the diffusers. The data used for the instantaneous flow analysis in Figs. 12 and 13 are extracted after 30 FTPs.

\section{Results and Discussion}

\subsection{Time-Averaged Flow}

The first- and second order statistics of the LES predictions are first validated with the Cherry et al. (2008) experimental and the Ohlsson et al. (2010) DNS data. In the current paper, the spatial and velocity conventions are consistent with previous studies on these diffusers, $(\mathrm{x}, \mathrm{u})$ represents the streamwise direction/velocity, $(\mathrm{y}, \mathrm{v})$ corresponds to the vertical direction/velocity and $(\mathrm{z}, \mathrm{w})$ denotes the spanwise direction/velocity.

Figure 5a, b respectively plot streamwise velocity profiles in the vertical planes located at $z / H=0.5$ and $z / H=7 / 8$ while Fig. 5c plots streamwise velocity profiles in the horizontal plane positioned at $y / H=1 / 8$. The Cherry et al. (2008) experimental data are only available for the vertical planes, however DNS-computed velocity profiles are available for all planes. The LES results of the five profiles along the centre plane (Fig. 5a) are all in excellent agreement with the experimental data. Peak velocities, flow reversal and boundary layer development predicted by the LES match well the experiment and are within the $5 \%$ error of the experimental data. In the second horizontal plane near the wall at $z / H=7 / 8$ (Fig. 5b) the computed time-averaged streamwise velocity profiles of D1 at $\mathrm{x} / \mathrm{H}=6$ and $\mathrm{x} / \mathrm{H}=10$ exhibit greater streamwise momentum than the experiment, this difference is probably the result of the near-wall resolution which is not as high than in the DNS. It is unlikely to be the result of the treatment of the corners in the numerical diffuser geometries because of the use of immersed boundaries in the expansion (note the experimental diffuser featured rounded-corners), because as can be seen at location $x / H=2$ the LES reproduces the onset of flow separation and the ensuing near wall velocity gradient rather well. Despite the slightly rounded corner in the experiment flow separation appears to occur immediately which is highlighted in Fig. 6a. Overall the LES velocity profiles at this location remain in fairly good agreement with the experimental data, accurately representing the size of the backflow region at $x / H=6, x / H=10$ and $x / H=15.5$. It is interesting to note that in both diffusers a clear deceleration of the high momentum core due to the adverse pressure gradient is observed reducing the core flow by $50 \%$ when reaching the station $\mathrm{x} / \mathrm{H}=18.5$. In Fig. 5a an inflection point is depicted in the mean streamwise velocity of $\mathrm{D} 1$ at $\mathrm{x} / \mathrm{H}=6$ evidencing the presence of strong shear flow and separated flow in its vicinity. Inflection points consist of localised loss of momentum in the velocity profile transferred from a nearby low-velocity region. This projection is validated as the flow separates at $\mathrm{x} / \mathrm{H}=10$ in $\mathrm{D} 1$, this phenomenon is also observable in D2 but to a lesser extent as it occurs at $\mathrm{x} / \mathrm{H}=10$ while exhibiting only a very small separated portion near the top wall at $\mathrm{x} / \mathrm{H}=15.5$. At location $\mathrm{z} / \mathrm{H}=7 / 8$ closer to the top right corner of the diffusers, the velocity profiles feature such inflection points much sooner at $\mathrm{x} / \mathrm{H}=2$ and both flows separate before $\mathrm{x} / \mathrm{H}=6$. It is interesting to observe that due to the steeper expansion of the top wall in D1 a stronger adverse pressure gradient is present, enforcing an earlier and larger separation of the flow compared to D2. It results in a shift of the core and a slight increase in the high momentum region of the flow in D1 demonstrating the higher blockage effect that ensues from the backflow region. Further, in the spanwise direction, see 

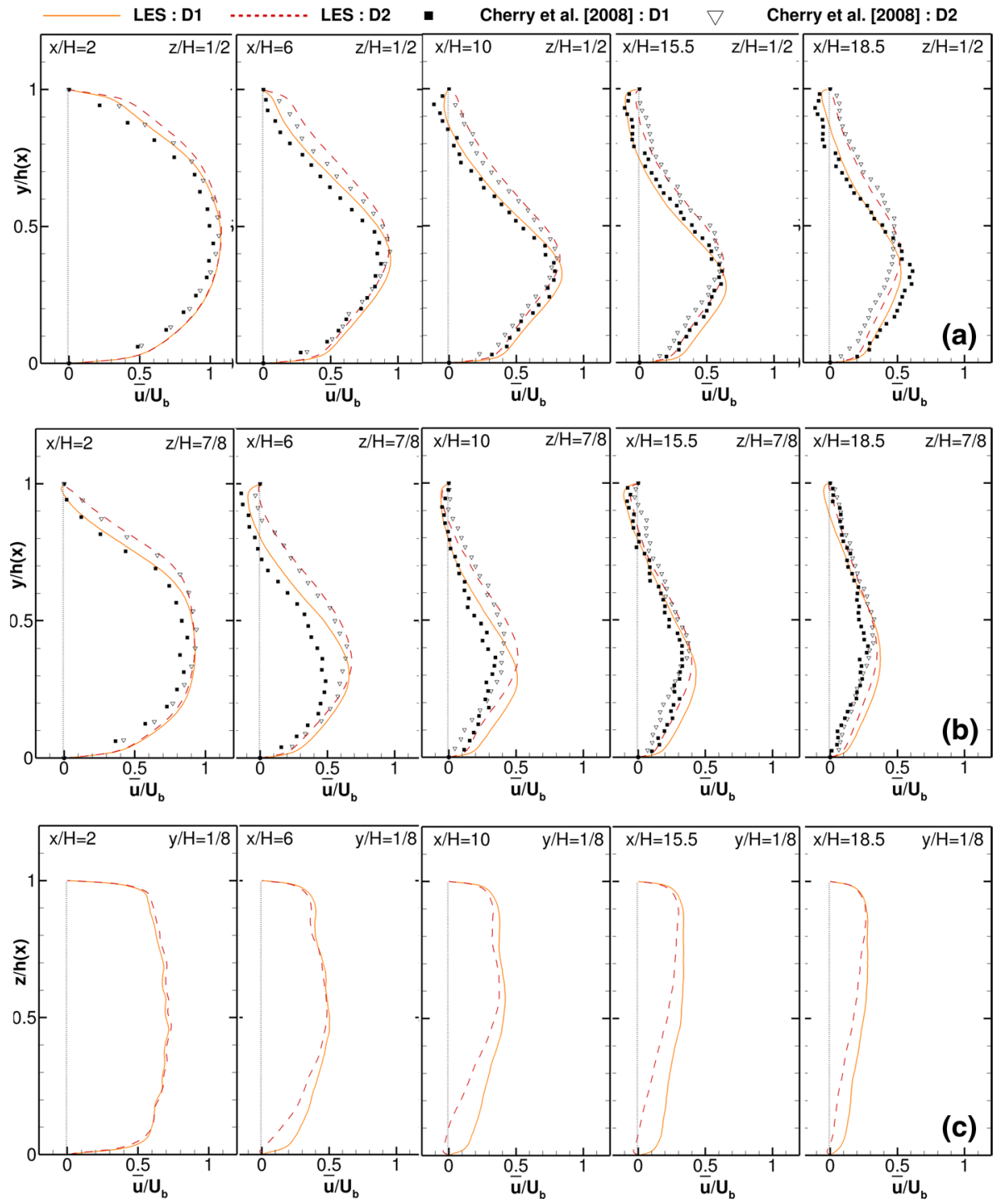

Fig. 5 Profiles of the normalised mean streamwise velocity $\bar{u} / U_{b}$ : in the a Vertical plane $\mathrm{z} / \mathrm{H}=1 / 2$, b Vertical plane $\mathrm{z} / \mathrm{H}=7 / 8$, and $\mathbf{c}$ horizontal plane $\mathrm{y} / \mathrm{H}=1 / 8$

Fig. 5c, D2 is endowed with a higher sidewall expansion revealing a recirculating zone from $\mathrm{x} / \mathrm{H}=6$ which seems to extend all the way to $\mathrm{x} / \mathrm{H}=18.5$.

Figure 6 presents an iso-surface of $\bar{u}=0.001$ of the mean streamwise velocity in both diffusers, D1 (a) and D2 (b), as well as blow-ups of the dominating flow separation in the upper right corners. The blow-ups highlight that flow separation occurs immediately after the flow enters the expansion while facing the adverse pressure gradient irrespective of diffuser geometry. However there is a clear disparity in terms of location and size of the timeaveraged backflow regions between the two diffusers. D1 features only one reverse flow 


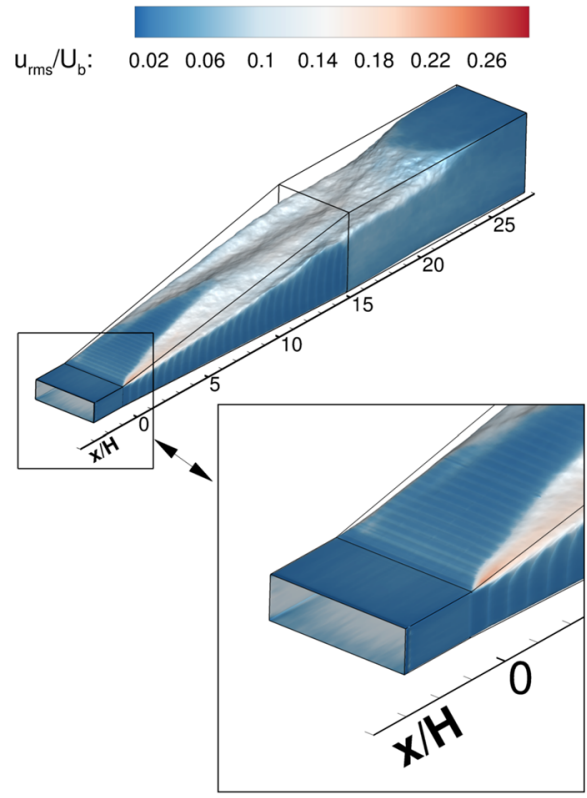

(a)

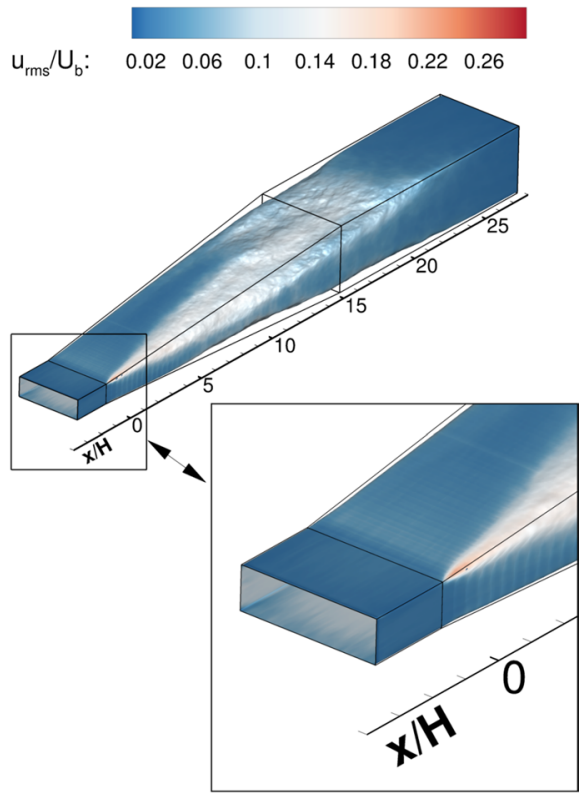

(b)

Fig. 6 Iso-surface of the Flow separation $(\bar{u}=0.001)$ with a normalised root mean square of the fluctuation contour $\left(u_{r m s} / U_{b}\right)$ : a D1, b D2

emerging at the throat of the diffuser. It originates in the corner of the top and side walls which induces an abrupt deceleration and growth of the boundary layer which eventually detaches from its wall(s). The backflow region quickly grows diagonally downstream until the middle of the diffuser where it occupies the full width of the diffuser, from that point on the flow separation may nearly be considered two-dimensional as it progresses quasiuniformly until its reattachment point at $x / H=23$. The second diffuser's mean velocity field displays two distinctively different recirculation regions, similarly to D1 the first recirculation zone originates from the asymmetric top corner at the outset of the expansion section propagating more gradually and diagonally across the full width of the diffuser's top wall before reattaching earlier than in $\mathrm{D} 1$ at $x / H=20$. The second mean backflow volume is much smaller than the first one, it emanates from the bottom right expanding corner at $x / h=6$ and reattaches at $x / H=22$. High levels of streamwise turbulence $\left(u_{r m s} / U_{b}\right)$ is present in both diffusers at the interface between mean reverse flow and the streamwise core flow. This characteristic is further examined and detailed in the next section.

Figure 7 presents LES-computed and measured contours of the normalised streamwise velocity in selected cross-sections for both diffusers. The figure demonstrates that in the D1 diffuser, the flow separation emerges from the top right expanding corner and grows nearly equally on either side of the diffuser $(x / H<5)$ before merging fully at the top wall $(\mathrm{x} / \mathrm{H}=12)$ where the separated flow displays quasi-two-dimensional flow separation characteristics. The LES is in very good agreement with the measurements except that the second backflow area seems to appear slightly earlier in the LES than in the experiment (at $\mathrm{x} / \mathrm{H}=8$ ). Although this mechanism is less pronounced in the results of Schneider et al. (2010) and Ohlsson et al. (2010), their simulations also display a 'bubble' at $\mathrm{x} / \mathrm{H}=8$. For the diffuser D2, the mean flow separation behaviour is mostly in good agreement with the 


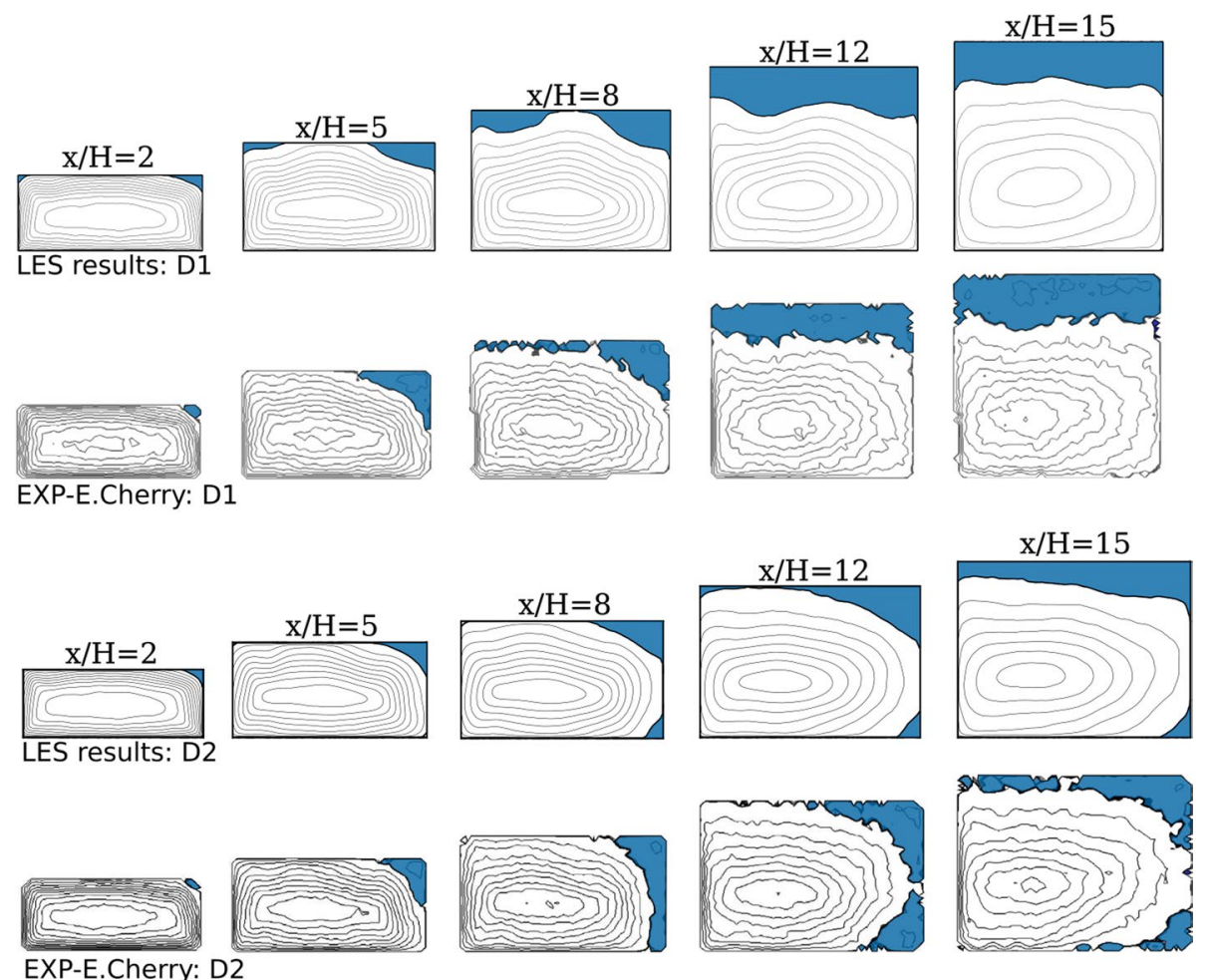

Fig. 7 Normalised mean streamwise velocity contour $\left(\bar{u} / U_{b}\right)$ of D1 and D2 at multiple cross-sections $(\mathrm{x} / \mathrm{H}=5,8,12,15)$. Contour lines spaced $0.1 \mathrm{~m} / \mathrm{s}$ apart, bold line represents $\bar{u} / U_{b}=0$, shaded area represents the backflow

experiment, there is a slight difference in the formation of the bottom right corner bubble, which in the experiment seems to occur somewhere between $\mathrm{x} / \mathrm{H}=5$ and $\mathrm{x} / \mathrm{H}=8$. In the LES results two distinctive bubbles form at the top right corner and bottom right corner while in the experiment the top right corner bubble spreads fully onto the sidewall $(\mathrm{x} / \mathrm{H}=8)$ before splitting up further downstream $(\mathrm{x} / \mathrm{H}=15)$ (Fig. 7).

The fractional area occupied by the mean reverse flow quantifies the blockage enforced onto the flow field of the diffuser and it is plotted as a function of distance from the entrance in Fig. 8. The gradient of the fractional area near the entrance of D1 is in excellent agreement with the experiment until approximately $\mathrm{x} / \mathrm{H}=10$ where the two recirculating regions converge and the LES overpredicts the magnitude of the backflow region by approximately $3 \%$. Although the gradient of the reduction of the separated flow is very similar to the experiment, the reattachment point is slightly delayed until $\mathrm{x} / \mathrm{H}=23$ in the LES simulation compared to $\mathrm{x} / \mathrm{H}=21$ in the experiment. Contrarily, the second diffuser D2 somewhat under-predicts the gradient of the fractional area translating to a smaller flow separation growth which delays its peak to $\mathrm{x} / \mathrm{H}=16$ compared to the experimental peak located at $\mathrm{x} / \mathrm{H}=12$. The reattachment point of the flow separation in both the experiment and simulation coincide at $\mathrm{x} / \mathrm{H}=21$. The hydrodynamics of three-dimensional flow separations are challenging to capture accurately both experimentally and computationally. Abe and Ohtsuka (2010) provides the experimental fractional area which accounts for 


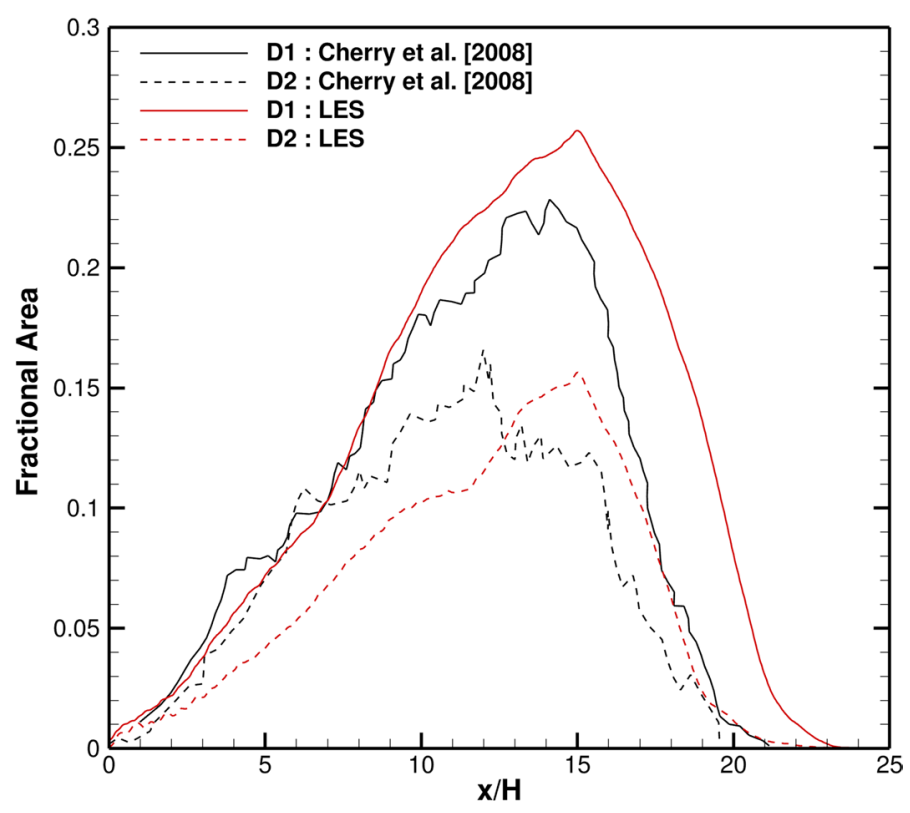

Fig. 8 Fractional area occupied by the mean flow separation within the rectangular diffusers: D1 and D2

instrumentation error margin, the spatial disparities of the LES results reside well within these error margins.

\subsection{Second Order Turbulence Statistics and Kinetic Energy}

Figure 9a presents profiles of the normalised root mean square of the streamwise velocity fluctuation $\left(u_{r m s} / U_{b}\right)$ at five locations $(\mathrm{x} / \mathrm{H}=2,6,10,15.5,18.5)$ of $\mathrm{D} 1$ and $\mathrm{D} 2$ along the centre vertical plane, i.e. $\mathrm{z} / \mathrm{H}=1 / 2$. The experimental data is only available for the streamwise intensity for D1. The LES results are overall in excellent agreement with the experimental data. The two diffusers share similar characteristics in the development of the streamwise turbulence intensity along the vertical slice of both diffusers. In the first section at $\mathrm{x} / \mathrm{H}=2$, the streamwise turbulence intensity presents a similar profile as the one found in the flow development duct, the high turbulence intensity zones are located both near the top wall and bottom wall of the diffusers while a low-intensity zone is found at half the height of the section within the core flow. Subsequently, the high shear stress zone at the bottom of the diffusers is slightly reduced along the expansion; further, the high shear stress peak located at the top of the diffusers is gradually shifted toward the middle of the sections' heights from $\mathrm{x} / \mathrm{H}=5$ to $\mathrm{x} / \mathrm{H}=18.5$. This is due to the development of the mean flow separation, for which the developing shear layer due to the contact of the no-slip wall and streamwise velocity is now transferred to the interface between the recirculation zone and the streamwise flow. The high streamwise intensity zone is of slightly smaller magnitude in D2 when compared to D1, it reflects the smaller portion of the top wall occupied by the mean flow separation in D2. 

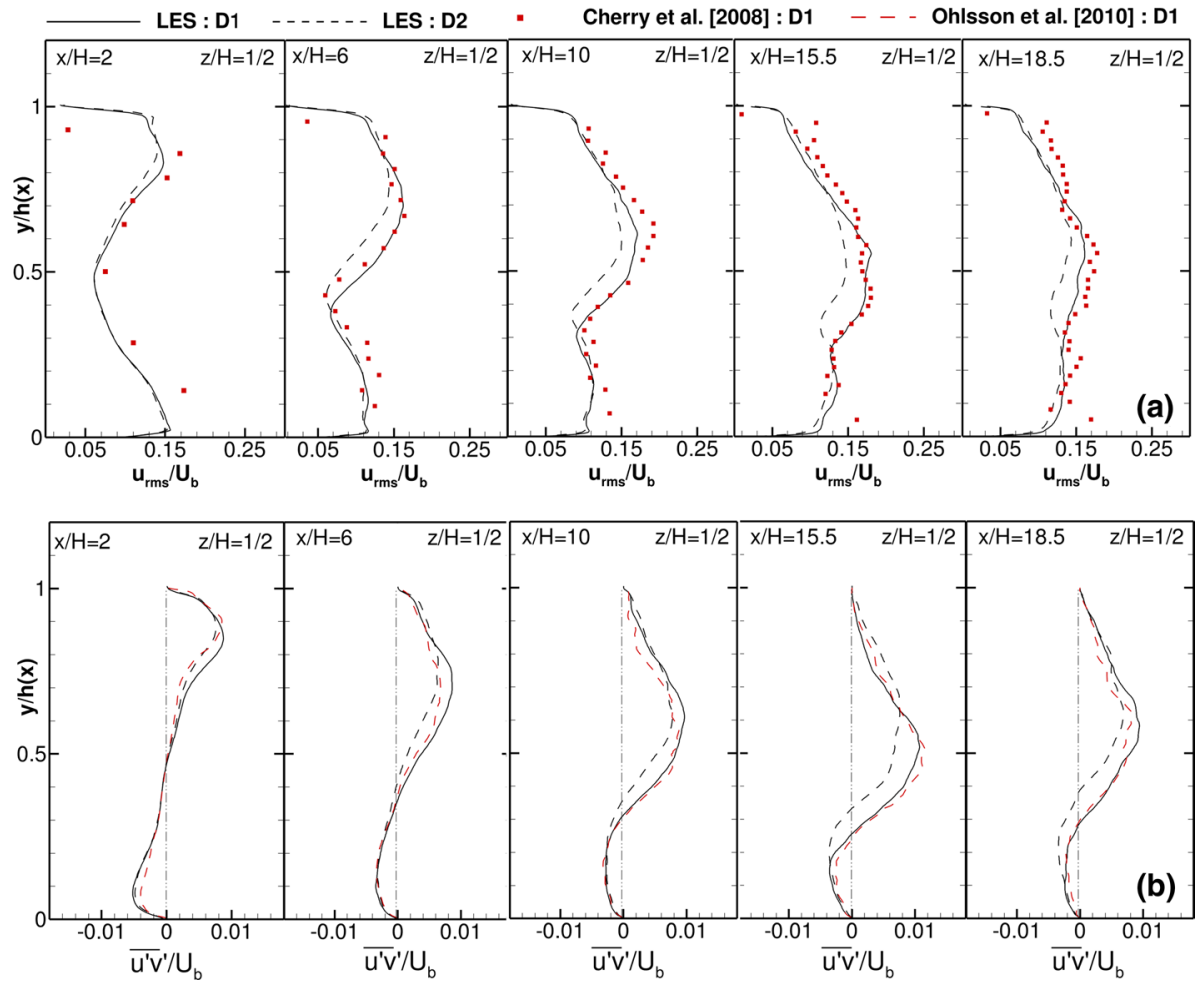

Fig. 9 Vertical profiles located on the vertical plane $\mathrm{z} / \mathrm{H}=1 / 2$ : a Normalised root mean square of the streamwise turbulent fluctuations $u_{r m s} / U_{b}$, and $\mathbf{b}$ normalised shear stress $\overline{u^{\prime} v^{\prime}} / U_{b}$

Profiles of the $\left\langle u^{\prime} v^{\prime}>/ U_{b}\right.$ shear stress in the centre line of the same five cross-sections of diffusers D1 and D2 are plotted in Fig. 9b. The LES profiles of D1 are in excellent agreement with the DNS data of Ohlsson et al. (2010). As expected, the shear stress profile is symmetric at the beginning of the diffuser exhibiting equivalent shear stress magnitude on both top and bottom walls. Similarly to the streamwise turbulence, the shear stress peaks increase in the downstream direction and gradually shift away from the top wall while the peaks remain constant near the bottom wall while only slightly shifting away. The profiles of the shear stresses are nearly identical in both diffusers, the smaller volume of the backflow near the top wall in D2 results in a slight vertical shift of the shear stress peak.

Figure 10 presents contours of the normalised turbulent kinetic energy (TKE) in crosssections at the same $\mathrm{x} / \mathrm{H}$ locations as the above profiles. At the beginning of the expansion, $\mathrm{x} / \mathrm{H}=2$, both diffusers exhibit a high kinetic energy zone in the top right corner and D1 also has a high TKE zone located at the top left corner heralding the development of separated flow at these locations. Major differences in the distribution of TKE between D1 and D2 are appreciated at $\mathrm{x} / \mathrm{H}=8$, where the TKE zone in D1 is larger and the magnitude of the TKE is greater than in D2. At $x / H=12$ the high TKE zone of D1 has shifted left of the centre and the peak TKE of D2 has already reduced somewhat. In both diffusers, the magnitude of the highest zone in each section decreases downstream, the highest $T K E / U_{b}^{2}$ magnitude zones are located in the first section of D1 (0.047) and D2 (0.04) while the peak magnitude in the last cross-section has reduced by $40 \%$ in D1 or by $30 \%$ in D2. 


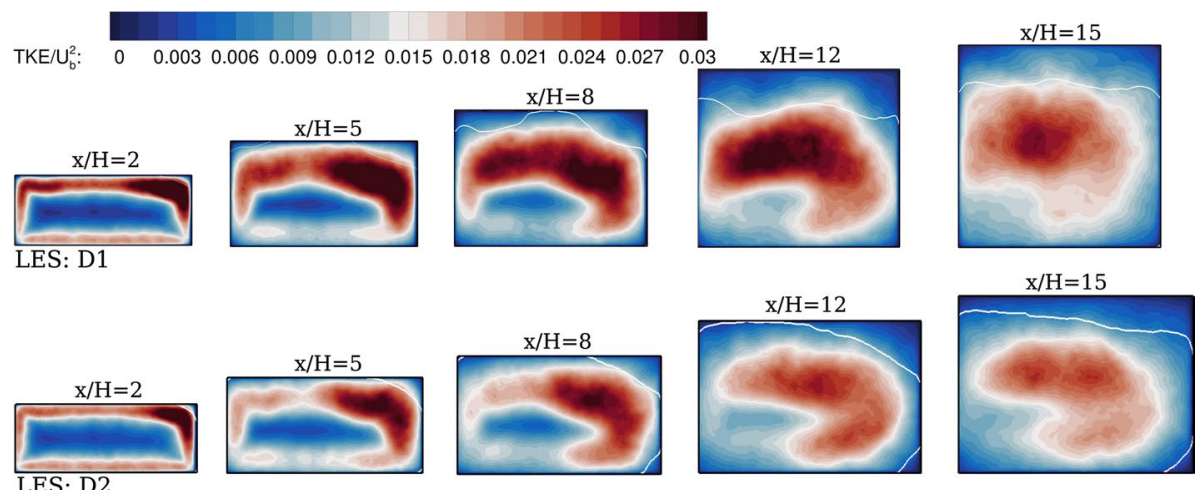

Fig. 10 Contours of the turbulent kinetic energy in multiple cross-sections $(x / H=5,8,12,15)$ along the D1 and D2 diffusers. The white line represents the mean zero-streamwise velocity $\bar{u}=0$

\subsection{Pressure Coefficient and Efficiency}

The pressure coefficient, $C_{P}$, is a long-established and key measurement in diffuser design to evaluate their performance and efficiency. $C_{P}$ represents the change of the static pressure normalised with the dynamic head at the diffuser inlet. Two distinctive methods exist: the first and most widely used is the one-dimensional pressure coefficient, it assesses the local pressure along the centerline near the wall of the diffuser while the second method, deemed more accurate and is used in this study, calculates the pressure coefficient using the pressure area average of each cross-section within the diffuser (Eq. 2). The efficiency and non-dimensional head losses are conveniently derived from the pressure coefficient. The efficiency $\left(\eta=C_{P R} / C_{P R i}\right)$ corresponds to the ratio between the measured pressure coefficient and the ideal pressure coefficient of uniform inviscid and frictionless flow $\left(C_{P R i}=1-1 /(A R)^{2}\right)$ while the non-dimensional head loss is the difference between the two aforementioned terms $\left(H_{L}=C_{P R i}-C_{P R}\right)$.

$$
C_{P R S}=\frac{\left(1 / A_{s}\right) \int P_{s} d A_{s}-\left(1 / A_{r e f}\right) \int P_{r e f} d A_{r e f}}{0.5 \rho\left(1 / A_{r e f}\right) \int U_{1}^{2} d A_{r e f}}
$$

Figure 11a presents the average pressure coefficient of each section along the diffuser. The LES slightly underpredicts the pressure recovery in the D1 diffuser after $\mathrm{x} / \mathrm{H}=10$ but is overall in very good agreement with the experimental data of Cherry et al. (2009). This slight difference is directly correlated with the size of the mean flow recirculation area. In both diffusers, a strong adverse pressure gradient develops between $x / H \in[0,2]$ near the throat of the diffuser before considerably reducing after $x / H>2$ once the mean flow separation starts to spread across the diffusers (Fig. 11b). Subsequently, the adverse pressure gradient follows similar trends to the growth gradient of the backflow fractional area in Fig. 8. The non-dimensional headloss $\left(H_{L}\right)$ reveals an inflection point at the interface between the end of the expansion part and the tail section of the diffuser $(x / H=15)$ from which the losses decrease inversely proportional to the pressure coefficient due to the reduction and dissipation in fractional area of the recirculating flow. Similar findings are observed in the planar diffuser of Törnblom et al. (2009). The efficiency of D2 is slightly 


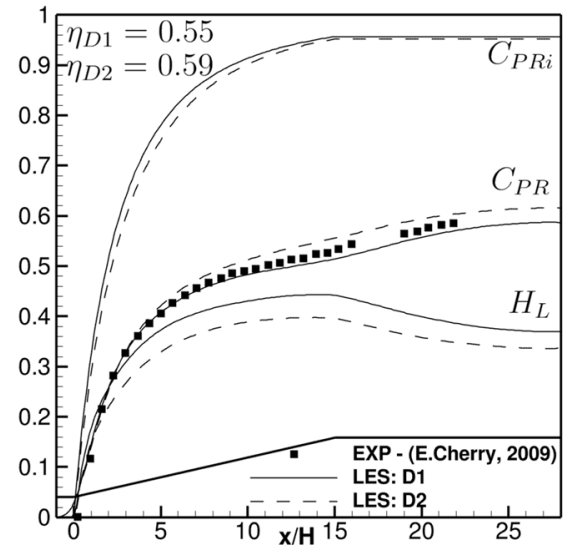

(a)

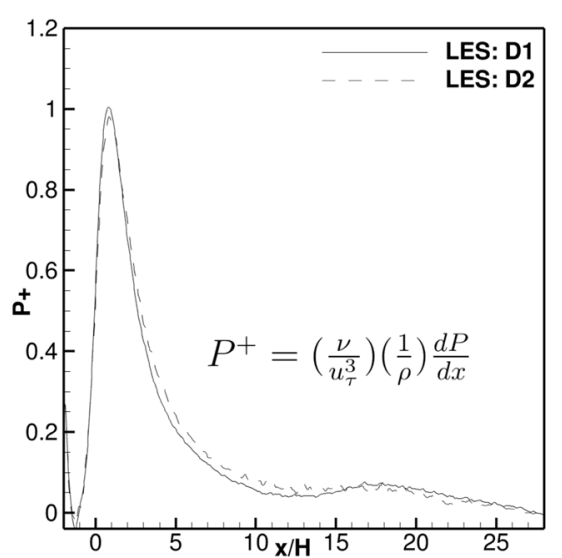

(b)

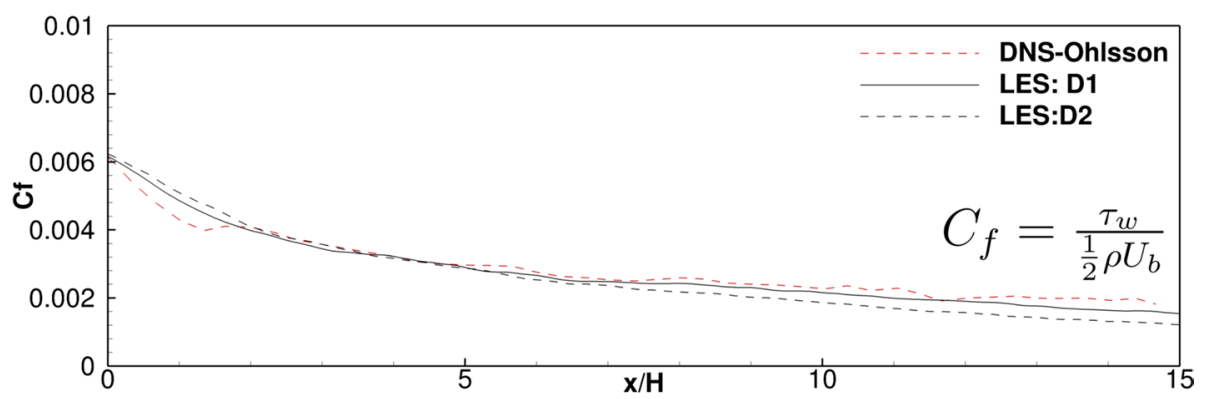

(c)

Fig. 11 a One-dimensional pressure coefficient $\mathrm{Cp}$, b Dimensionless pressure gradient $\mathrm{P}+$, $\mathbf{c}$ Friction Coefficient $\mathrm{Cf}$ along the bottom centerline of the two rectangular diffusers

higher than D1 with values of $59 \%$ or $55 \%$, respectively. The centreline profile of the coefficient of friction is plotted in Fig. 11, and evidently the LES-predicted Cf is in fairly good agreement with the DNS Results from Ohlsson et al. (2010). Similarly to the pressure gradient (Fig. 11b), there is only a slight difference in the profiles between D1 and D2, suggesting that the difference in AER between the two diffusers does not affect significantly the behaviour of the flow separation.

\subsection{Instantaneous Flow}

Prior to the quantification of the temporal unsteadiness of the backflow region, the unsteadiness of the backflow inside each of the two diffusers is visualised. Figures 12 and 13 provide contours of the instantaneous streamwise velocity at three different instants in time in the $\mathrm{X}-\mathrm{Y}$ plane along $\mathrm{z} / \mathrm{H}=7 / 8$ and the $\mathrm{X}-\mathrm{Z}$ plane at $\mathrm{y} / \mathrm{H}=1 / 8$. The instantaneous flow in both diffusers does not exhibit a single large recirculation zone as suggested in Fig. 6, but instead, the meandering of the flow entering the diffuser leads to a multitude of high shear stress zones close to the wall that in turn forces the streamwise flow to separate from its turbulent boundary layer and to reverse. The snapshots highlight specific locations of the separated flow and the behaviour is perceived from visualisation and animation 

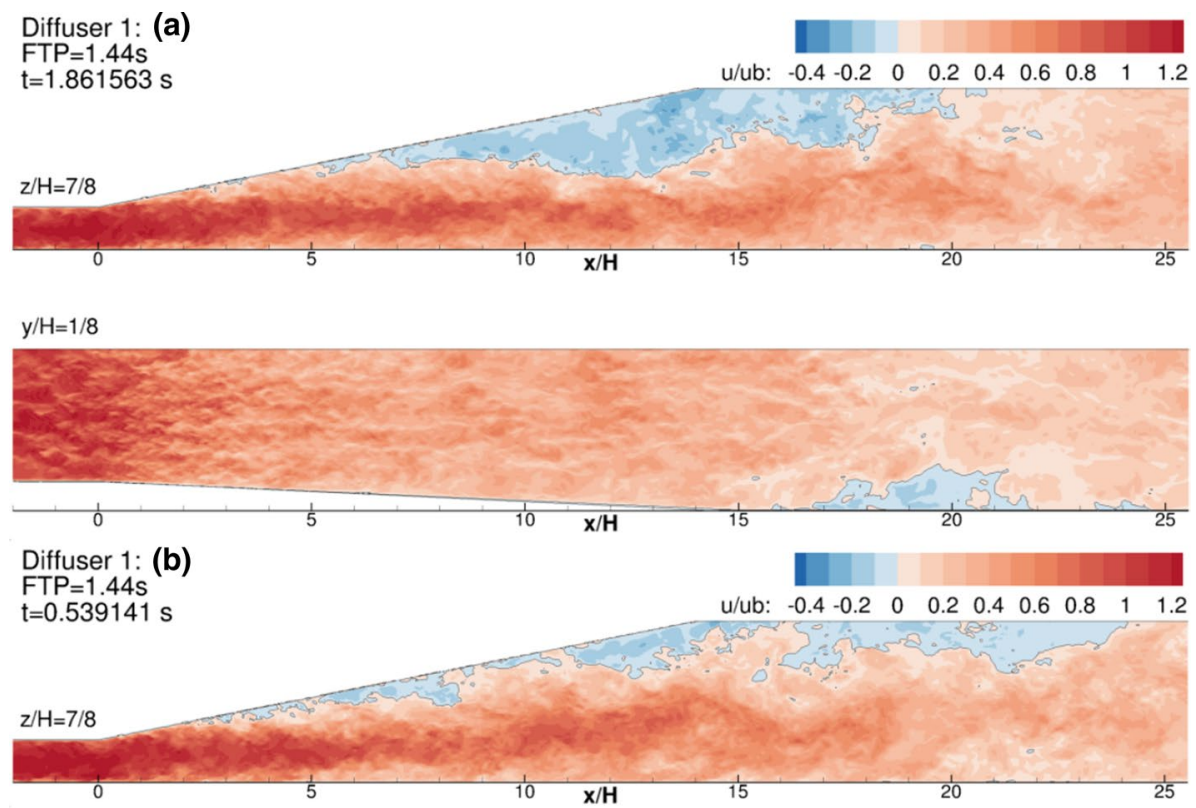

$y / H=1 / 8$
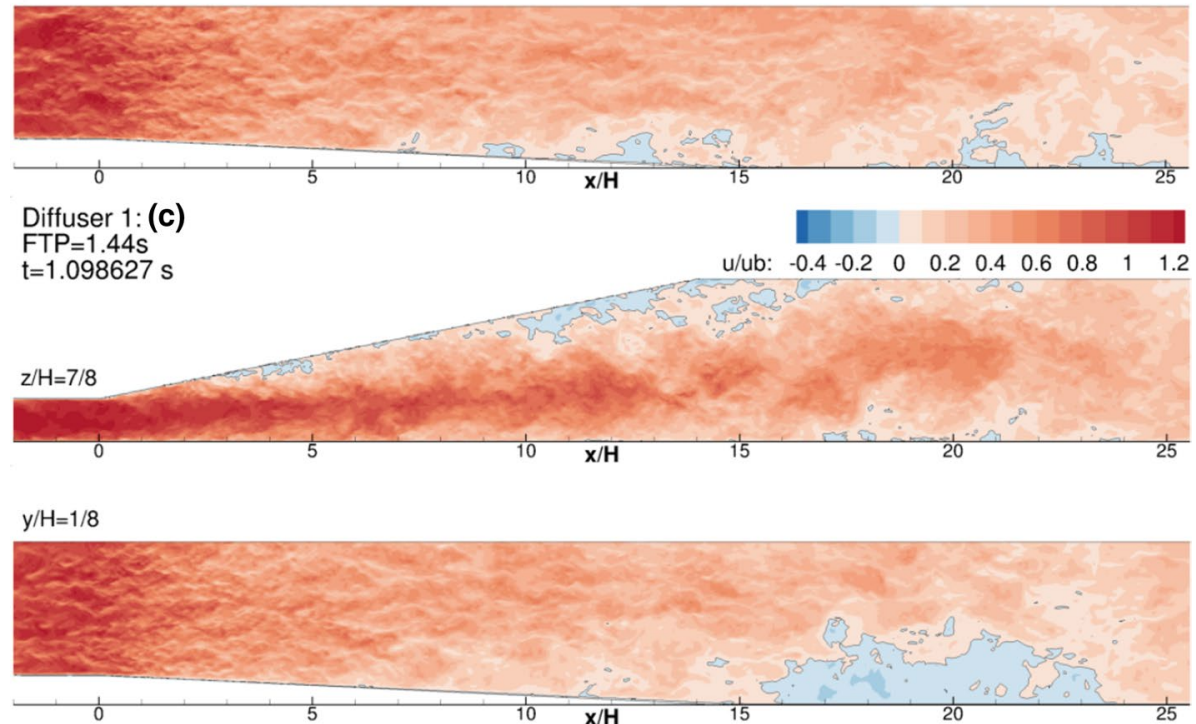

Fig. 12 D1: Contours of the normalised instantaneous streamwise velocity $u / U_{b}$ in cross-sectional and longitudinal near-wall planes at three selected instants in time: $\mathrm{t}=0.54,1.1,1.86$

of more than 1000 snapshots representing 10 FTPs through both diffusers. These reveal a core meandering generated through the constant action-reaction feedback between the streamwise high-velocity core and the different reverse flow pockets. In Fig. 13b, the recirculation zone located at $\mathrm{x} / \mathrm{H}=10$ is easily distinguished from the core velocity near the 

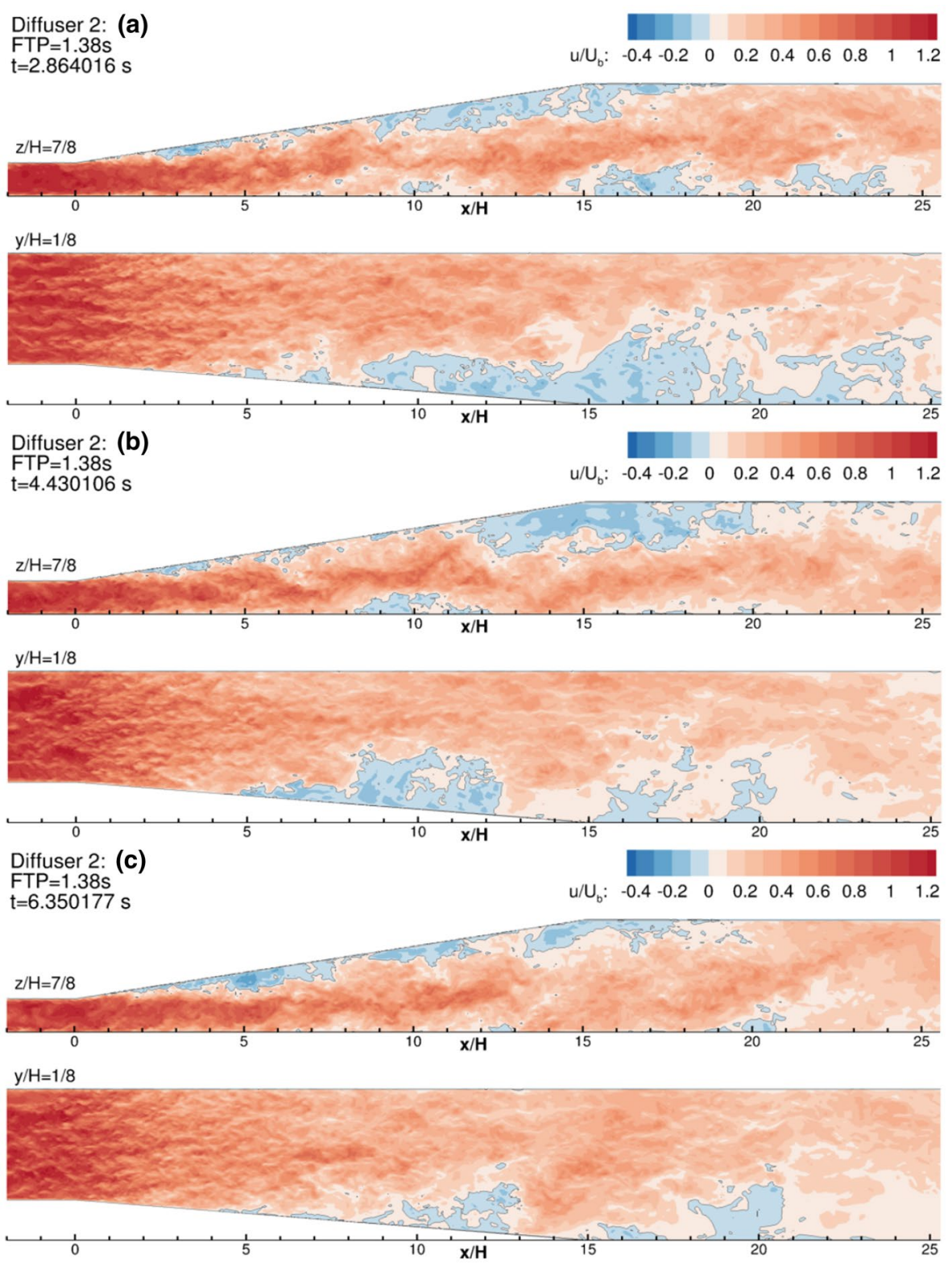

Fig. 13 D2: Contours of the normalised instantaneous streamwise velocity $u / U_{b}$ in cross-sectional and longitudinal near-wall planes at three selected instants in time: $\mathrm{t}=2.86,4.43,6.35$

top inclined wall. Although, the meandering phenomenon is present in both diffusers, it is evident from the respective snapshots that the aspect ratio results in the flow separating at different locations.

The large-angle expansion in D1 drives the instantaneous flow to separate away from the boundary layer of the top inclined wall. The backflow pockets contain high reverse 
momentum attempting to travel upstream toward the throat of the diffuser (Fig. 12a). These pockets often stagnate around $\mathrm{x} / \mathrm{H}=5$ and $\mathrm{x} / \mathrm{H}=15$ and are only occasionally washed away before dissipating on their way towards the end of the diffuser whilst forming large reverse flow zones as seen in Fig. 12b. Near diffuser D1's wall, reverse flow significantly detaches at $\mathrm{x} / \mathrm{H}=10$ and is subsequently transported toward the end of the diffuser to form a large instantaneous backflow region with low kinetic energy. The separated flow located at the top wall of diffuser D1 dictates the meandering of the core.

The behaviour of flow separation and interaction with the core flow is slightly different in the second diffuser, D2, its lower asymmetric expansion ratio $A E R_{D 2}=2$ results in the development of significant reverse flow on both expanding walls (Fig. 12a). Similarly to diffuser D1, the reverse flow pockets travel towards the throat of the diffuser (Fig. 13c), but remarkably in $\mathrm{D} 2$, stationary pockets form both on the top and side expansion walls around $\mathrm{x} / \mathrm{H}=10$ (Fig. 13b).

\subsection{Unsteadiness of the Flow Separation}

The backflow coefficient $(\gamma)$ is an excellent indicator for quantifying the flow separation unsteadiness, Simpson (1996) first introduced the method which consists of computing the period during which the flow moves in the streamwise direction. A backflow coefficient of $\gamma=1$ corresponds to a region where no instantaneous flow reversal is recorded during the simulation, inversely if $\gamma=0$ the region only comprises reverse flow. Simpson (1996) classifies the unsteadiness of a region in three categories, $\gamma=0.99$ corresponds to an Incipient Detachment (ID), $\gamma=0.8$ satisfies an Intermittent Transitory Detachment (ITD) and $\gamma=0.5$ represents a Transitory Detachment (TD) equivalent to mean flow separation results in Fig. 6. The current classification does not include the location of reversal of high momentum pockets within the diffuser. In an effort to complement and harmonise the current classification one could consider $\gamma=0.2$ as Persistent Transitory Detachment (PTD) and $\gamma=0.01$ as Permanent Detachment (PD).

Table 2 complements Figs. 14 and 15 to enable the quantification and location of these quasi-permanent stagnation zones and other classified unsteadiness regions. The $2 \mathrm{D}$ contours of the backflow coefficient suggest that the ID region is similar in both diffusers but
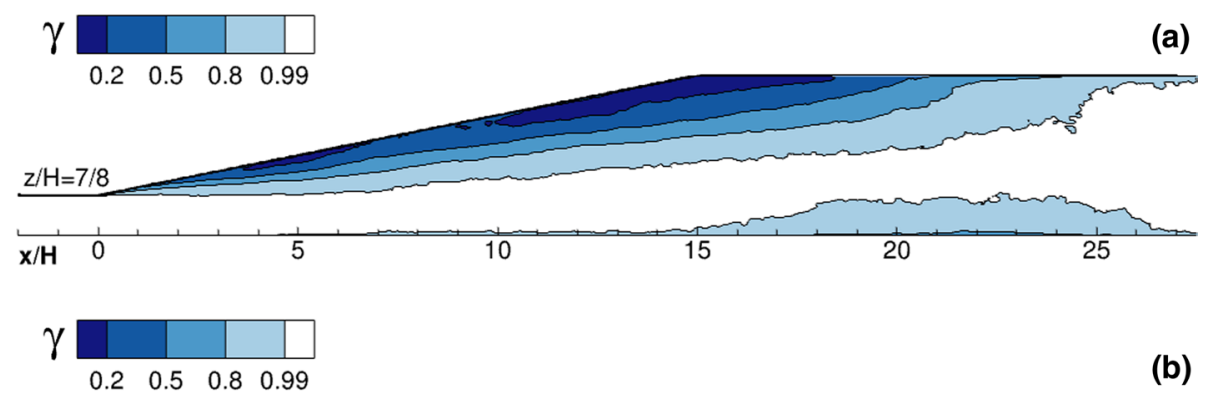

(b)

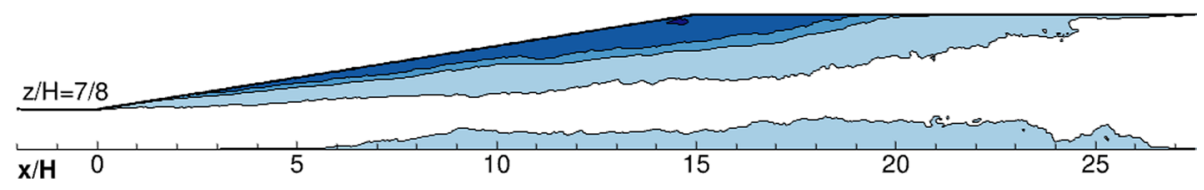

Fig. 14 Contours of the backflow coefficient in the longitudinal near-wall plane at $\mathrm{z} / \mathrm{H}=7 / 8$ : a D1, b D2 

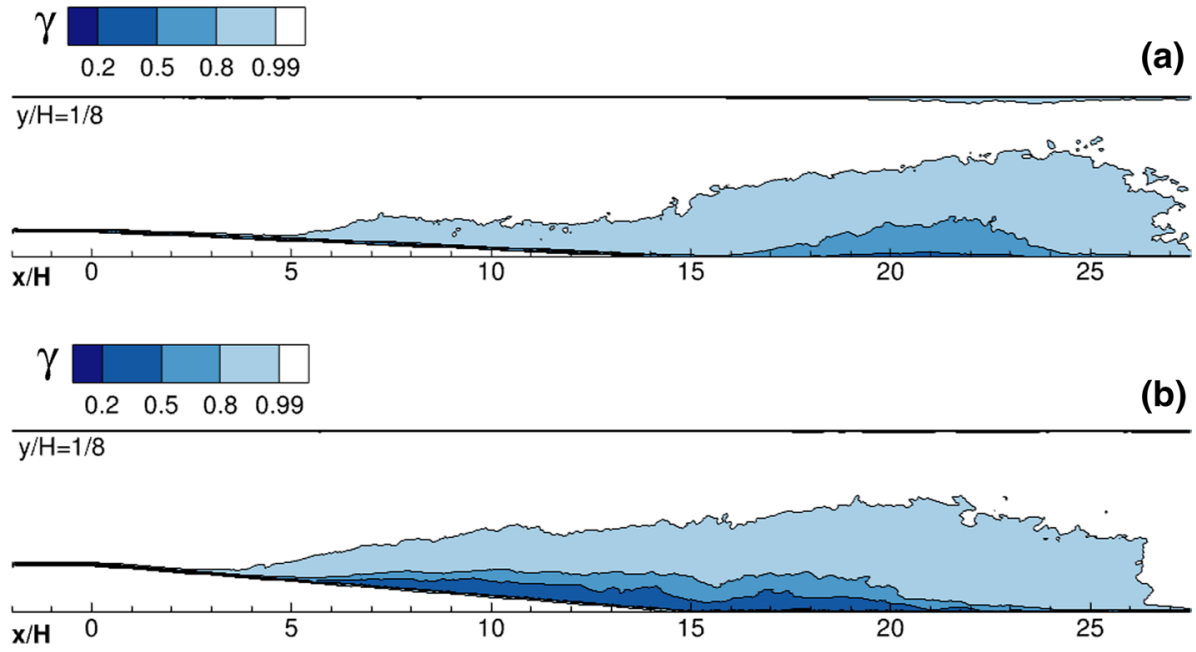

Fig. 15 Contours of the backflow coefficient in the horizontal near-wall plane at $y / H=1 / 8$ : a D1, b D2

Table 2 Fractional volume in terms of the backflow coefficients: $\gamma=0.99,0.5,0.2$ within diffusers D1 and D2

\begin{tabular}{llll}
\hline FS Type & D1:FV1 $(\%)$ & D1:FV2 $(\%)$ & D1:FVT (\%) \\
\hline ID $(\gamma=0.99)$ & 48.03 & 50.69 & 49.58 \\
TD $(\gamma=0.5)$ & 16.33 & 8.12 & 11.55 \\
PTD $(\gamma=0.2)$ & 6.98 & 3.18 & 4.75 \\
\hline FS Type & D2:FV1 $(\%)$ & D2:FV2 $(\%)$ & D2:FVT (\%) \\
\hline ID $(\gamma=0.99)$ & 42.15 & 37.79 & 39.59 \\
TD $(\gamma=0.5)$ & 9.20 & 3.27 & 5.71 \\
PTD $(\gamma=0.2)$ & 2.00 & 0.21 & 0.97 \\
\hline
\end{tabular}

$I D$ incipient detachment, $T D$ transitory detachment, $P T D$ persistent transitory detachment

Table 2 reveals that the fractional volume of this region is $10 \%$ greater in D1(49.6\%) than in D2(39.6\%). This ID region describes the delimitation volume in with all the reversal separated flow emerges, develops and dissipates. The mean flow separation (TD) is inevitably larger in D1(11.55\%) than in $\mathrm{D} 2(5.71 \%)$ and the respective location of these zones are in line with previous observations (from Fig. 6), the D1 TD zone is much larger than in D2 in Fig. 14, inversely D2 TD zone expands between $x / H \in[5,23]$ while the D1 TD is tiny between $x / H \in[18,22]$ in Fig. 15. The PTD region is depicted at two different locations in D1 vertical plane between $x / H \in[3.5,7]$ and $x / H \in[10,17.5]$ while only a tiny zone around $\mathrm{x} / \mathrm{H}=15$ is present in $\mathrm{D} 2$, no PTD regions are observed in the horizontal plane (Fig. 15). The fractional volume occupied by the PTD region within D1(4.95\%) is nearly 5 times larger than in D2(0.97\%) further explaining the greater drop in efficiency of D1 as these regions act as long-lasting localised blockage effect within diffusers. In Cherry et al. (2008) the fractional volume of the mean flow separation within the expansion part of the diffuser (FV1) is examined and the LES data of D1(16.33\%) and D2 $(9.20 \%)$ are in 
relatively good agreement with the experimental data of both diffuser respective $14.1 \%$ and $10.7 \%$. It is interesting to note the importance of the tail section (FV2) specifically in diffuser design as both flow separation zones recover in this section. The volume occupied by the mean flow separation in the tail section is halved in D1 in comparison with D2 and nearly three times smaller in D2 which exhibits a much faster recovery.

Figure 16a presents the temporal evolution of the cumulative instantaneous separated flow normalised by the cumulative volume of both expansion and extension sections of the two diffusers. It calculates the ratio between cells having flow reversal (negative streamwise velocity) and the total number of cells constituting the expansion and extension sections, i.e. the volume in percentage of the backflow region will be of smaller magnitude than previously. The mean cumulative backflow volumes are $11.4 \%$ and $8.1 \%$ of the D1 and D2 volumes, respectively. The diffusers' time series both exhibit a clear phase of growth followed by a reduction phase of the total instantaneous separated flow. The Fast Fourier Transformation of the time series fluctuations (Fig. 16b) clearly captures these distinctive phases in both diffusers. The growth phase has a non-dimensional periodicity (Strouhal number) of $S t=0.7$ while the reduction phase periodicity is $S t=0.1$. It is interesting to note that at no point in time the reverse flow fully disappears. For diffuser D1, the cumulative backflow volume ranges from 7 to $13.94 \%$ while for D2 it fluctuates between 5.1 and $12 \%$. The root mean square values which evaluate the level of fluctuation around the mean value of the time series suggests that the flow separation is more unsteady in D2 than in D1 having values of $B V_{r m s}=14.3 \%$ (D2) and $B V_{r m s}=9.8 \%$ (D1). It suggests that a high level of unsteadiness is beneficial toward improving the efficiency of diffusers as it prevents the development of Persistent Transitory Detachment.

\subsection{Turbulent Boundary Layer and Coherent Flow Structures}

The instantaneous flow separation emerges from high instantaneous shear regions near the walls of the diffusers, which are manifested through the formation of energy-containing motions, also called coherent structures. The topology and classification of these coherent structures remain challenging for experimentalists due to their unsteadiness, scale and versatility. The method of quadrant analysis was first developed by Wallace
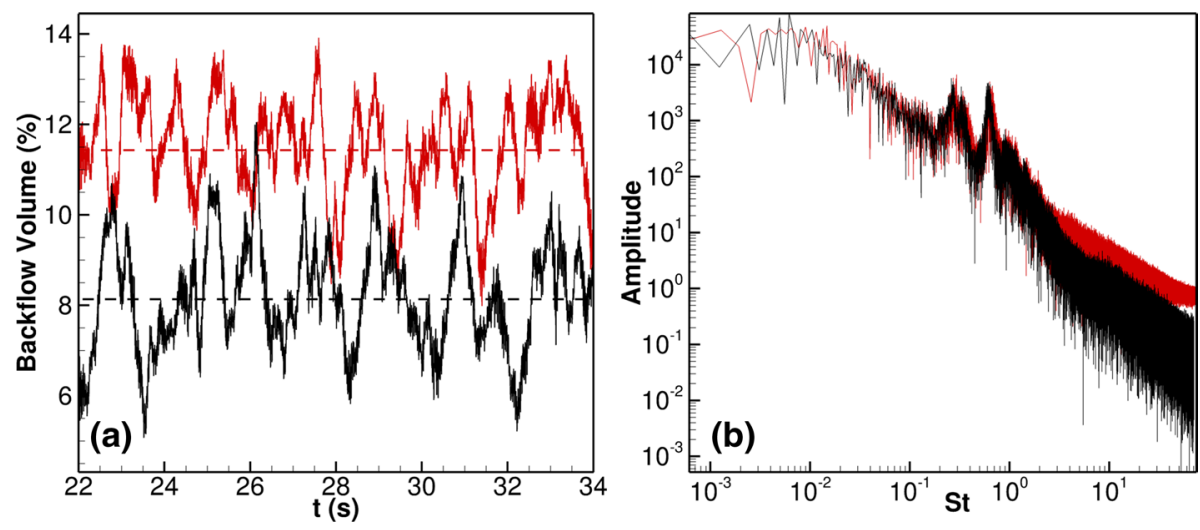

Fig. 16 a Flow separation total volume time series across expansion and extension sections of the two rectangular diffusers and $\mathbf{b}$ PSD of the flow separation total volume series 
et al. (1972) to reveal the presence of energy-containing motions in a boundary layer and was soon adopted to investigate near-wall turbulence of open-channel flow (Kim et al. 1987; Zhou et al. 1999). The quadrant analysis relies on a time series of the velocity fluctuations comprising the anisotropic Reynolds Stresses at specified probe locations. Quadrant one $\left(u^{\prime}>0, w^{\prime}>0\right)$ reveals a forward and upward motion (also referred to as outward interaction. The second quadrant $\left(u^{\prime}<0, w^{\prime}>0\right)$ describes a backward and upward motion, referred to as ejection, while the third quadrant $\left(u^{\prime}<0, w^{\prime}<0\right)$ causes a backward and downward momentum, or inward interaction. The fourth quadrant $\left(u^{\prime}>0, w^{\prime}<0\right)$ describes a forward and downward motion, known as a sweep. In the two diffusers, all directions are bounded by smooth walls depending on the probe location the motion is analysed relative to its closest wall to understand if the fluid is breaking away from the wall toward the core of the flow or if it is conveying towards the wall.

In order to identify, visualise and examine coherent structures in the diffuser flow, velocity probes are placed in the core of the high momentum zone, in the vicinity of the top and bottom wall and near the boundary of the time-averaged flow separation zone. The probes' locations are denoted from P1 to P9 in Fig. 19. Quadrant analysis is carried out using a matrix of $100 \times 100$ using 300,000 data pairs for each of the nine points within the two diffusers (Wallace and Brodkey 1977). It is equivalent to a computational time of D1 $=14$ FTPs and D2 = 15 FTPs. The most centre and smallest contour correspond to the maximum occurrence event $(=1)$; subsequently, each line contour is spaced with 0.1 increments. For instance, the turbulent events located in between the third and second line contour have an occurrence of $80 \%$ compared to the first contour. It enables better visualisation of the distribution and density of the events.

Figures 17 and 18 plot the normalised streamwise $\left(u^{\prime} / u_{r m s}\right)$ and vertical $\left(v^{\prime} / v_{r m s}\right)$ fluctuations at these 9 locations which are spread over the $\mathrm{z} / \mathrm{H}=1 / 2$ plane. The nature of the turbulent motion is evaluated at 3 different sections, near the entrance of the diffuser $\mathrm{x} / \mathrm{H}=2(\mathrm{P} 1, \mathrm{P} 2, \mathrm{P} 3)$, in the middle of the expansion section at $\mathrm{x} / \mathrm{H}=10(\mathrm{P} 4, \mathrm{P} 5, \mathrm{P} 6)$ and within the tail section at $\mathrm{x} / \mathrm{H}=20(\mathrm{P} 7, \mathrm{P} 8, \mathrm{P} 9)$. Three probes are respectively placed near the bottom wall, near the top wall and in the middle of the section. In all three sections, the probes located near the top wall and the bottom present similar quadrant characteristics in both diffusers. The motion near the bottom wall P1, P4, P7 possess similar temporal coherence and share dominating Q2 (sweep) and Q4 (ejection) events, suggesting the occurrence of hairpin vortices from the flat bottom wall of the diffusers (Adrian 2007). Near the top inclined wall, the primary motions are forward and upward (Q1) and backward and downward (Q3). These motions are not characteristic of the development of hairpin vortices (Zhou et al. 1999) when the referenced wall is located below the probe. However, in this specific case, the inclined wall is located above the probe inverting the quadrant mechanism, the Q1 corresponds to a forward upward motion toward the wall (sweep) while Q3 is backward downward motion away from the wall. These motions at P3, P6 and P9, are attributed to the presence of coherent structures near the top inclined wall. In both diffusers, evidently, the distribution at P2 located in the core flow is more isotropic and the Reynolds shear stresses are homogeneous. It is the region of the lowest shear hence without the development and conveying of significant turbulence structures. Further, after reaching the end of the expansion section the meandering core flow has dissipated. At P7, P8 and P9, the quadrant analysis captures the energy-containing motions that are convected from the top and bottom wall of the expansion part of the diffuser (see Fig. 19). The sweep motion towards the wall is the dominant turbulence event of the probes located near the top (P1, P4) or bottom wall (P3, P6) of the diffusers' expansion section. 

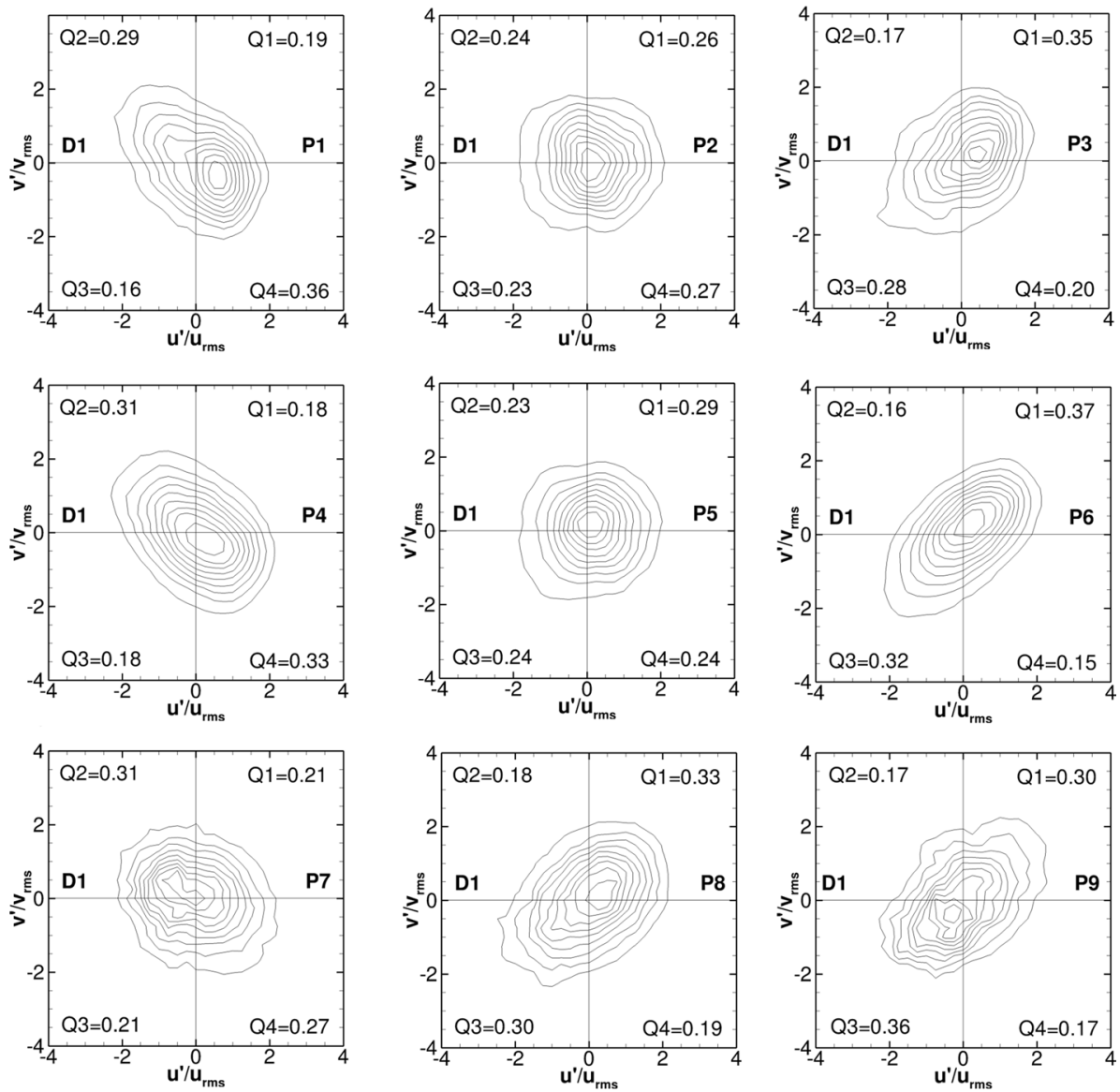

Fig. 17 Quadrant analysis of the shear stress u'v' at specific locations in diffuser D1

The quadrant analysis does provide not only information regarding the formation of energy-containing motion but also reflects on the state of the flow. In the middle probe of the second section, P5 is more isotropic in D1 than in D2 but both respectively have sweep dominant quadrants $\mathrm{Q} 1=29$ and $\mathrm{Q} 1=35$. The location and dominance of sweeps at this location suggest that sweeps towards the top inclined wall that acts as an opposing force to the reversal flow interface attempting a reattachment. The two diffusers display some discrepancies nonetheless, at P6 the sweep motions are dominant in D1 (Q1 = 37) while ejection events are prominent in $\mathrm{D} 2(\mathrm{Q} 3=39)$. In the tail section, the third quadrant Q3 is clearly dominant in P9 $(\mathrm{Q} 3: \mathrm{D} 1=0.36, \mathrm{D} 2=0.37)$ heralding the presence of recirculating flow at these locations. Near the bottom wall at P7, the turbulence events are relatively isotropic in D2 while strong ejection events remain in D1 $(\mathrm{Q} 3=31)$ which may be due to the development of ITD during the monitoring of that probe.

Experimental investigations mostly rely on quadrant analysis, or 2D-planes, to depict the characteristic motions of coherent structures in the vicinity of the turbulent boundary layer. Numerical investigations possess a significant visualisation advantage enabling the classification and revelation of the topology of the coherent structures. The Q criterion is 

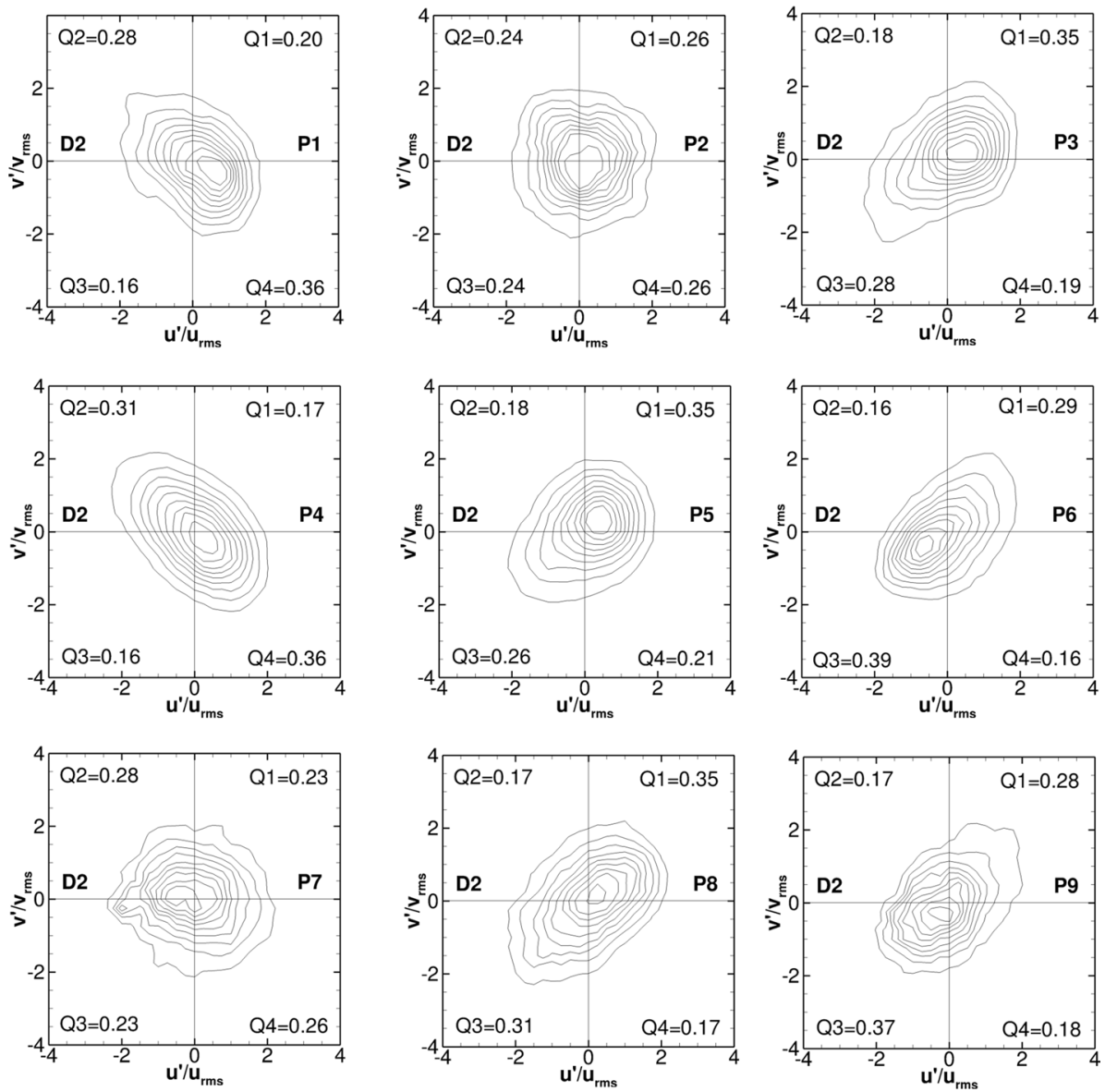

Fig. 18 Quadrant analysis of the shear stress u'v' at specific locations in diffuser D2

the difference between the symmetric (Strain rate $=$ Strain rate $=S_{i k} S_{k j}$ ) and the anti-symmetric (Rotation rate $=\Omega_{i k} \Omega_{k j}$ ) part of the velocity gradient tensor of $u_{i, j}$. If Q is positive, the coherent structures are dominated by rotation, when $\mathrm{Q}$ is negative, the energy-containing motion is inversely driven by strain. The coherent structures illustrated in Fig. 19 presenting isosurfaces of $\mathrm{Q}=75,000$, are only composed of small scale turbulent eddies and large structures are absent from the flow field. One can depict two distinctive phenomena, the eddies bursting from the top turbulent boundary layer $(x / H \in[0,5])$ and their conveyance to the tail section through a passage bounded by the core flow and the separated flow, while the coherent structures emerging from the bottom turbulent boundary layer are constrained by the wall and the core flow. The core flow dissipates around $\mathrm{x} / \mathrm{H}=15$, the energy-containing structures from the top and bottom wall mix together and is convected by the streamwise flow. An isometric view of the $\mathrm{Q}$ criterion three-dimensional visualisation reveals the emergence and conveying of quasi-streamwise vortices including lowspeed streaks, high-speed streaks and short spanwise hairpin vortices when conveyed away from the wall. The presence of these quasi-streamwise hairpins is also supported by Figs. 12 and $13 \mathrm{y} / \mathrm{H}=1 / 8$ contour. These contours exhibit high-momentum and low 


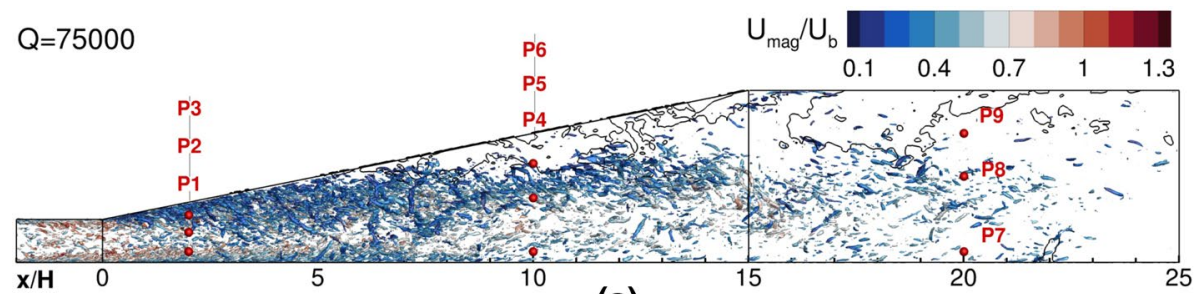

(a)

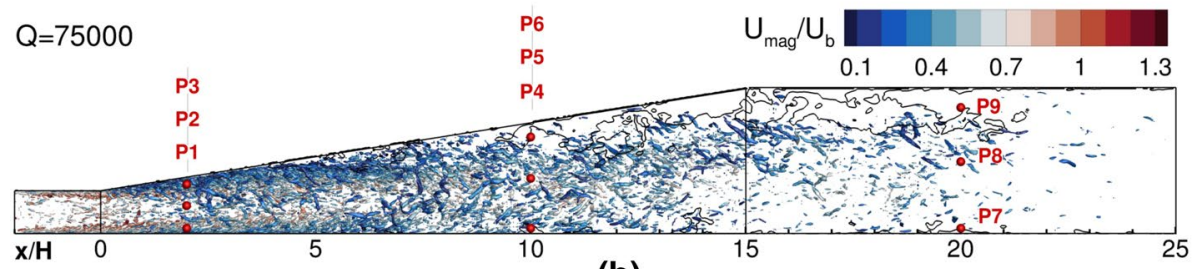

(b)

Fig. 19 Iso-surface of the Q-Criterion $(\mathrm{Q}=75,000)$ contoured with the normalised Velocity magnitude $\left(U_{m a g} / U_{b}\right)$ : a D1, b D2

momentum streaks between $x / H \in[-2,5]$ which is characteristic by these quasi-streamwise hairpins (Tomkins and Adrian 1999). Malm et al. (2012) arrived at similar findings with a POD analysis, which did not depict any large horseshoes vortices. The observed coherent structures can be considered as vorticity transporting entities (Adrian 2007), supposedly the meandering of the core flow does not provide enough stability in the high shear region to sustain the development of large horseshoes vortices.

\section{Conclusions}

Large-eddy simulations of flow in two asymmetric diffusers (D1 and D2) have been performed. The accuracy and quality of the LES in terms of the time-averaged flow and its fluctuations have been confirmed by comparing computed with measured data of Cherry et al. (2008) where good agreement was achieved. After successful validation of the simulations, the efficiency and pressure recovery of the two diffusers were evaluated. The mean recirculation zones in the diffuser are bounded by high shear stress and subsequently regions of elevated turbulent kinetic energy (TKE). The second diffuser with an area aspect ratio of 2.01 shows better performance in terms of pressure loss and efficiency than the first diffuser with an area aspect ratio of 4.46. The hydrodynamic behaviour of the instantaneous flow separation in both diffusers has been described through visualisation of the instantaneous flow in vertical and horizontal planes at selected instants in time. The quasi-periodic behaviour of the flow separation consisting of numerous smaller pockets of reverse flow near the top and side expanding walls, which travel upstream toward the throat of the diffuser, has been shown via contour plots of the instantaneous velocity. Depending on the size and the velocity magnitude of the reverse flow, these pockets either dissipate or merge with other reverse flow pockets to form larger recirculation bubbles. Whilst in diffuser D2 these large recirculation pockets are washed away quite rapidly by the meandering core flow, some of these large backflow regions stagnate persistently in D1 and are hence being 
considered Persistent Transitory Detachment (PTD). The PTD pockets reduce the unsteadiness of the flow significantly creating long-lasting local flow blockage, thereby reducing greatly the deceleration rate of the incoming flow, which translates into higher pressure losses and less efficient pressure recovery. The Power Spectral Density (PSD) of the fractional volume of the instantaneous reversal flow time series revealed a growth and reduction phase of the instantaneous flow separation. The two diffusers exhibit similar growth $(\mathrm{St}=0.7)$ and reduction $(\mathrm{St}=0.1)$ phases suggesting a gradual accumulation of reversal flow during the growth followed by a rapid detachment and downwash of the large recirculation zones. The root mean square of this time series evaluates the unsteadiness of the flow separation and quantifies the PTD as D1 $B V_{r m s}=9.8 \%$ and D2 $B V_{r m s}=14.3 \%$. It suggests that high unsteadiness in the flow separation leads to improvement in efficiency and performance of the diffuser. Lastly, quadrant analyses at various locations inside D1 and D2 have revealed the characteristics of the shear stress propitious to the emergence of coherent structures. The Q criterion iso-surface of the flow depicts the occurrence of low-speed streak, high-speed streaks and small spanwise rollers within the flow field of both diffusers.

Acknowledgements The authors would like to acknowledge the support of the Supercomputing Wales project, which is part-funded by the European Regional Development Fund (ERDF) via the Welsh Government.

Funding This work was supported by the Engineering and Physical Sciences Research Council in the UK via Grant EP/L016214/1 awarded for the Water Informatics: Science and Engineering (WISE) Centre for Doctoral Training, which is gratefully acknowledged.

Data Availability The LES data of the D2 diffuser is available at: Arthur Hajaali, Thorsten Stoesser. (2021). Large Eddy Simulation data of flow in a rectangular diffuser [Data set]. Zenodo. https://doi.org/10.5281/ zenodo. 5624237 .

\section{Declarations}

Conflict of Interest The authors have no financial or proprietary interests in any material discussed in this article.

Open Access This article is licensed under a Creative Commons Attribution 4.0 International License, which permits use, sharing, adaptation, distribution and reproduction in any medium or format, as long as you give appropriate credit to the original author(s) and the source, provide a link to the Creative Commons licence, and indicate if changes were made. The images or other third party material in this article are included in the article's Creative Commons licence, unless indicated otherwise in a credit line to the material. If material is not included in the article's Creative Commons licence and your intended use is not permitted by statutory regulation or exceeds the permitted use, you will need to obtain permission directly from the copyright holder. To view a copy of this licence, visit http://creativecommons.org/licenses/by/4.0/.

\section{References}

Abe, K., Ohtsuka, T.: An investigation of LES and Hybrid LES/RANS models for predicting 3-D diffuser flow. Int. J. Heat Fluid Flow 31(5), 833-844 (2010). https://doi.org/10.1016/j.ijheatfluidflow.2010.03. 005

Adrian, R.J.: Hairpin vortex organization in wall turbulence. Phys. Fluids 19, 041301 (2007)

Azevedo, J., Durst, F., Pereira, J.: Comparison of strongly implicit procedures for the solution of the fluid flow equations in finite difference form. Appl. Math. Model. 12(1), 51-62 (1988). https://doi.org/10. 1016/0307-904X(88)90023-6

Bomminayuni, S., Stoesser, T.: Turbulence statistics in an open-channel flow over a rough bed. J. Hydraul. Eng. 137(11), 1347-1358 (2011). https://doi.org/10.1061/(ASCE)HY.1943-7900.0000454

Cevheri, M., McSherry, R., Stoesser, T.: A local mesh refinement approach for large-eddy simulations of turbulent flows. Int. J. Numer. Methods Fluids 82(5), 261-285 (2016). https://doi.org/10.1002/fld.4217 
Cherry, E.M., Elkins, C.J., Eaton, J.K.: Geometric sensitivity of three-dimensional separated flows. Int. J. Heat Fluid Flow 29(3), 803-811 (2008). https://doi.org/10.1016/j.ijheatfluidflow.2008.01.018

Cherry, E.M., Elkins, C.J., Eaton, J.K.: Pressure measurements in a three-dimensional separated diffuser. Int. J. Heat Fluid Flow 30(1), 1-2 (2009). https://doi.org/10.1016/j.ijheatfluidflow.2008.10.003

Cherry, E.M., Padilla, A.M., Elkins, C.J., Eaton, J.K.: Three-dimensional velocity measurements in annular diffuser segments including the effects of upstream strut wakes. Int. J. Heat Fluid Flow 31(4), 569-575 (2010). https://doi.org/10.1016/j.ijheatfluidflow.2010.02.029

Chorin, A.J.: Numerical solution of the Navier-Stokes equations. Math. Comput. 22(104):745-762, iSBN: 0025-5718 (1968)

Daniels, S., Rahat, A., Tabor, G., Fieldsend, J., Everson, R.: Shape optimisation of the sharp-heeled Kaplan draft tube: performance evaluation using computational fluid dynamics. Renew. Energy 160, 112-126 (2020). https://doi.org/10.1016/j.renene.2020.05.164

Duquesne, P., Maciel, Y., Deschênes, C.: Investigation of flow separation in a diffuser of a bulb turbine. J. Fluids Eng. 138(1), 011102 (2016). https://doi.org/10.1115/1.4031254

Fishenden, C.R., Stevens, S.J.: Performance of annular combustor-dump diffusers. J. Aircr. 14(1), 60-67 (1977). https://doi.org/10.2514/3.58749

Gibson, A.H.: On the flow of water through pipes and passages having converging or diverging boundaries. Proc. R. Soc. A Math. Phys. Eng. Sci. 83(563), 366-378 (1910). https://doi.org/10.1098/rspa. 1910.0025

Grundmann, S., Sayles, E.L., Elkins, C.J., Eaton, J.K.: Sensitivity of an asymmetric 3D diffuser to vortex-generator induced inlet condition perturbations. Exp. Fluids 52(1), 11-21 (2012). https://doi. org/10.1007/s00348-011-1205-0

Herbst, A.H., Schlatter, P., Henningson, D.S.: Simulations of turbulent flow in a plane asymmetric diffuser. Flow Turbul. Combust. 79(3), 275-306 (2007). https://doi.org/10.1007/s10494-007-9091-5

Jakirlić, S., Kadavelil, G., Kornhaas, M., Schäfer, M., Sternel, D.C., Tropea, C.: Numerical and physical aspects in LES and hybrid LES/RANS of turbulent flow separation in a 3-D diffuser. Int. J. Heat Fluid Flow 31(5), 820-832 (2010). https://doi.org/10.1016/j.ijheatfluidflow.2010.05.004

Kara, M.C., Stoesser, T., McSherry, R.: Calculation of fluid-structure interaction: methods, refinements, applications. Proc. Inst. Civ. Eng. Eng. Comput. Mech. 168, 59-78 (2015)

Kim, J., Moin, P., Moser, R.: Turbulence statistics in fully developed channel flow at low Reynolds number. J. Fluid Mech. 177, 133-166 (1987). https://doi.org/10.1017/S0022112087000892

Kline, S.: Optimum Design of Straight Walled Diffusers. ASME (1959)

Laliberte, G.E., Shearer, M.N., English, M.J.: Desing of energy-efficient pipe-size expansion. J. Irrigation Drain. Eng. 109(1), 13-28 (1983). https://doi.org/10.1061/(ASCE)0733-9437(1983)109:1(13)

Malm, J., Schlatter, P., Henningson, D.S.: Coherent structures and dominant frequencies in a turbulent three-dimensional diffuser. J. Fluid Mech. 699, 320-351 (2012). https://doi.org/10.1017/jfm.2012. 107

McSherry, R.J., Chua, K.V., Stoesser, T.: Large eddy simulation of free-surface flows. J. Hydrodyn. 29(1), 1-12 (2017). https://doi.org/10.1016/S1001-6058(16)60712-6

McSherry, R.J., Chua, K.V., Stoesser, T., Mulahasan, S.: Free surface flow over square bars at intermediate relative submergence. J. Hydraul. Res. 56(6), 825-843 (2018). https://doi.org/10.1080/00221 686.2017.1413601

Nicoud, F., Ducros, F.: Subgrid-scale stress modelling based on the square of the velocity gradient tensor. Flow Turbul. Combust. 62(3), 183-200 (1999). https://doi.org/10.1023/A:1009995426001

Obi, S., Aoki, K., Masuda, S.: Experimental and computational study of turbulent separating flow in an asymmetric plane diffuser. In: Ninth Symposium on Turbulent Shear Flows, pp. 305-1. Hyoto, Japan (1993)

Obi, S., Nikaido, H., Masuda, S.: Reynolds Number Effect on the Turbulent Separating Flow in an Asymmetric Plane Diffuser, vol. 7. ASME (1999)

Ohlsson, J., Schlatter, P., Fischer, P.F., Henningson, D.S.: Direct numerical simulation of separated flow in a three-dimensional diffuser. J. Fluid Mech. 650, 307-318 (2010). https://doi.org/10.1017/S0022 112010000558

Ouro, P., Stoesser, T.: An immersed boundary-based large-eddy simulation approach to predict the performance of vertical axis tidal turbines. Comput. Fluids 152, 74-87 (2017). https://doi.org/10. 1016/j.compfluid.2017.04.003

Ouro, P., Stoesser, T.: Impact of environmental turbulence on the performance and loadings of a tidal stream turbine. Flow Turbul. Combust. 102, 613-639 (2019)

Ouro, P., Fraga, B., Lopez-Novoa, U., Stoesser, T.: Scalability of an Eulerian-Lagrangian large-eddy simulation solver with hybrid MPI/OpenMP parallelisation. Comput. Fluids 179, 123-136 (2019). https://doi.org/10.1016/j.compfluid.2018.10.013 
Patterson, G.N.: Modern Diffuser Design. AIRCRAFT ENGINEERING, p 7 (1938)

Schneider, H., von Terzi, D., Bauer, H.J., Rodi, W.: Reliable and accurate prediction of three-dimensional separation in asymmetric diffusers using large-eddy simulation. J. Fluids Eng. 132, 031101 (2010)

Simpson, R.L.: Aspects of turbulent boundary-layer separation. Prog. Aerosp. Sci. 32(5), 457-521 (1996). https://doi.org/10.1016/0376-0421(95)00012-7

Stoesser, T., McSherry, R., Fraga, B.: Secondary currents and turbulence over a non-uniformly roughened open-channel bed. Water 7(12), 4896-4913 (2015). https://doi.org/10.3390/w7094896

Tomkins, C.D., Adrian, R.J.: Characteristic of vortex packets in wall turbulence. In: First Symposium on Turbulence and Shear Flow Phenomena (1999)

Törnblom, O., Lindgren, B., Johansson, A.V.: The separating flow in a plane asymmetric diffuser with $\$ 8.5^{\wedge}\{$ circ $\} \$$ opening angle: mean flow and turbulence statistics, temporal behaviour and flow structures. J. Fluid Mech. 636, 337-370 (2009). https://doi.org/10.1017/S0022112009007940

Uhlmann, M.: An immersed boundary method with direct forcing for the simulation of particulate flows. J. Comput. Phys. 209(2), 448-476 (2005). https://doi.org/10.1016/j.jcp.2005.03.017.. arXiv:1809.08170

Wallace, J.M., Brodkey, R.S.: Reynolds stress and joint probability density distributions in the u-v plane of a turbulent channel flow. Phys. Fluids 20(3), 351 (1977). https://doi.org/10.1063/1.861897

Wallace, J.M., Eckelmann, H., Brodkey, R.S.: The wall region in turbulent shear flow. J. Fluid Mech. 54(1), 39-48 (1972). https://doi.org/10.1017/S0022112072000515

Welsh, M.: Flow control in wide-angled conical diffusers. ASME (1976)

Wu, X., Schlüter, J., Moin, P., Pitsch, H., Iaccarino, G., Ham, F.: Computational study on the internal layer in a diffuser. J. Fluid Mech. 550(1), 391 (2006)

Zhang, R., Mao, F., Liu, S.H.: Characteristics and control of the draft-tube flow in part-load Francis turbine. J. Fluids Eng. (2009). https://doi.org/10.1115/1.3002318

Zhou, J., Adrian, R.J., Balachandar, S., Kendall, T.M.: Mechanisms for generating coherent packets of hairpin vortices in channel flow. J. Fluid Mech. 387, 353-396 (1999). https://doi.org/10.1017/S002211209 900467X 